\title{
System of variational inequalities and an accretive operator in Banach spaces
}

\author{
Lu-Chuan Ceng ${ }^{1}$ and Ching-Feng Wen ${ }^{2 *}$
}

${ }^{\text {"Correspondence: }}$

cfwen@kmu.edu.tw

${ }^{2}$ Center for Fundamental Science, Kaohsiung Medical University,

Kaohsiung, 807, Taiwan

Full list of author information is

available at the end of the article

\begin{abstract}
In this paper, we introduce composite Mann iteration methods for a general system of variational inequalities with solutions being also common fixed points of a countable family of nonexpansive mappings and zeros of an accretive operator in real smooth Banach spaces. Here, the composite Mann iteration methods are based on Korpelevich's extragradient method, viscosity approximation method and the Mann iteration method. We first consider and analyze a composite Mann iterative algorithm in the setting of uniformly convex and 2-uniformly smooth Banach space, and then another composite Mann iterative algorithm in a uniformly convex Banach space having a uniformly Gâteaux differentiable norm. Under suitable assumptions, we derive some strong convergence theorems. The results presented in this paper improve, extend, supplement and develop the corresponding results announced in the earlier and very recent literature.

MSC: 49J30; 47H09; 47J20
\end{abstract}

Keywords: composite Mann iteration methods; general system of variational inequalities; accretive operator; nonexpansive mapping; sunny nonexpansive retraction; fixed point; uniformly Gâteaux differentiable norm; uniform smoothness; uniform convexity

\section{Introduction}

Let $X$ be a real Banach space whose dual space is denoted by $X^{*}$. The normalized duality mapping $J: X \rightarrow 2^{X^{*}}$ is defined by

$$
J(x)=\left\{x^{*} \in X^{*}:\left\langle x, x^{*}\right\rangle=\|x\|^{2}=\left\|x^{*}\right\|^{2}\right\}, \quad \forall x \in X,
$$

where $\langle\cdot, \cdot\rangle$ denotes the generalized duality pairing. It is an immediate consequence of the Hahn-Banach theorem that $J(x)$ is nonempty for each $x \in X$. Let $U=\{x \in X:\|x\|=1\}$ denote the unit sphere of $X$. A Banach space $X$ is said to be uniformly convex if for each $\epsilon \in(0,2]$, there exists $\delta>0$ such that for all $x, y \in U$,

$$
\|x-y\| \geq \epsilon \quad \Rightarrow \quad\|x+y\| / 2 \leq 1-\delta .
$$

It is known that a uniformly convex Banach space is reflexive and strict convex. A Banach space $X$ is said to be smooth if the limit

$$
\lim _{t \rightarrow 0} \frac{\|x+t y\|-\|x\|}{t}
$$

O2013 Ceng and Wen; licensee Springer. This is an Open Access article distributed under the terms of the Creative Commons Attribution License (http://creativecommons.org/licenses/by/2.0), which permits unrestricted use, distribution, and reproduction in any medium, provided the original work is properly cited. 
exists for all $x, y \in U$; in this case, $X$ is also said to have a Gâteaux differentiable norm. $X$ is said to have a uniformly Gâteaux differentiable norm if for each $y \in U$, the limit is attained uniformly for $x \in U$. Moreover, it is said to be uniformly smooth if this limit is attained uniformly for $x, y \in U$. The norm of $X$ is said to be the Frechet differential if for each $x \in U$, this limit is attained uniformly for $y \in U$. Let $C$ be a nonempty closed convex subset of $X$. A mapping $T: C \rightarrow C$ is called nonexpansive if $\|T x-T y\| \leq\|x-y\|$ for every $x, y \in C$. The set of fixed points of $T$ is denoted by $\operatorname{Fix}(T)$. A mapping $A: C \rightarrow X$ is said to be accretive if for each $x, y \in C$, there exists $j(x-y) \in J(x-y)$ such that $\langle A x-A y, j(x-y)\rangle \geq 0$.

Recently, Yao et al. [1] combined the viscosity approximation method and the Mann iteration method, and gave the following hybrid viscosity approximation method:

Let $C$ be a nonempty closed convex subset of a real uniformly smooth Banach space $X$, $T: C \rightarrow C$ a nonexpansive mapping such that $\operatorname{Fix}(T) \neq \emptyset$ and $f \in \Xi_{C}$ with a contractive coefficient $\rho \in(0,1)$, where $\Xi_{C}$ is the set of all contractive self-mappings on $C$. For an arbitrary $x_{0} \in C$, define $\left\{x_{n}\right\}$ in the following way:

$$
\left\{\begin{array}{l}
y_{n}=\alpha_{n} x_{n}+\left(1-\alpha_{n}\right) T x_{n}, \\
x_{n+1}=\beta_{n} f\left(x_{n}\right)+\left(1-\beta_{n}\right) y_{n}, \quad \forall n \geq 0,
\end{array}\right.
$$

where $\left\{\alpha_{n}\right\}$ and $\left\{\beta_{n}\right\}$ are two sequences in $(0,1)$. They proved under certain control conditions on the sequences $\left\{\alpha_{n}\right\}$ and $\left\{\beta_{n}\right\}$ that $\left\{x_{n}\right\}$ converges strongly to a fixed point of $T$. Subsequently, Ceng and Yao [2] under the convergence of no parameter sequences to zero proved that the sequence $\left\{x_{n}\right\}$ generated by (YCY) converges strongly to a fixed point of $T$. Such a result includes $[1$, Theorem 1$]$ as a special case.

Theorem 1.1 (See [2, Theorem 3.1]) Let $C$ be a nonempty closed convex subset of a uniformly smooth Banach space X. Let $T: C \rightarrow C$ be a nonexpansive mapping with $\operatorname{Fix}(T) \neq \emptyset$ and $f \in \Xi_{C}$ with contractive coefficient $\rho \in(0,1)$. Given sequences $\left\{\alpha_{n}\right\}$ and $\left\{\beta_{n}\right\}$ in $[0,1]$, the following control conditions are satisfied:

(i) $1 \leq \beta_{n} \leq 1-\rho, \forall n \geq n_{0}$ for some integer $n_{0} \geq 1$;

(ii) $\sum_{n=0}^{\infty} \beta_{n}=\infty$;

(iii) $0<\liminf _{n \rightarrow \infty} \alpha_{n} \leq \limsup _{n \rightarrow \infty} \alpha_{n}<1$;

(iv) $\lim _{n \rightarrow \infty}\left(\frac{\left.\beta_{n+1}\right) \alpha_{n+1}}{1-\left(1-\beta_{n+1}\right) \alpha_{n+1}}-\frac{\beta_{n}}{1-\left(1-\beta_{n}\right) \alpha_{n}}\right)=0$.

For an arbitrary $x_{0} \in C$, let $\left\{x_{n}\right\}$ be generated by $(\mathrm{YCY})$. Then

$$
x_{n} \rightarrow q \Longleftrightarrow \beta_{n}\left(f\left(x_{n}\right)-x_{n}\right) \rightarrow 0,
$$

where $q \in \operatorname{Fix}(T)$ solves the variational inequality problem $(V I P)$ :

$$
\langle q-f(q), J(q-p)\rangle \leq 0, \quad \forall p \in \operatorname{Fix}(T)
$$

On the other hand, $\mathrm{Cai}$ and $\mathrm{Bu}$ [3] considered the following general system of variational inequalities (GSVI) in a real smooth Banach space $X$, which involves finding $\left(x^{*}, y^{*}\right) \in$ $C \times C$ such that

$$
\begin{cases}\left\langle\mu_{1} B_{1} y^{*}+x^{*}-y^{*}, J\left(x-x^{*}\right)\right\rangle \geq 0, & \forall x \in C, \\ \left\langle\mu_{2} B_{2} x^{*}+y^{*}-x^{*}, J\left(x-y^{*}\right)\right\rangle \geq 0, & \forall x \in C,\end{cases}
$$


where $C$ is a nonempty, closed and convex subset of $X, B_{1}, B_{2}: C \rightarrow X$ are two nonlinear mappings and $\mu_{1}$ and $\mu_{2}$ are two positive constants. Here, the set of solutions of GSVI (1.1) is denoted by $\operatorname{GSVI}\left(C, B_{1}, B_{2}\right)$. In particular, if $X=H$ in a real Hilbert space, then GSVI (1.1) reduces to the following GSVI of finding $\left(x^{*}, y^{*}\right) \in C \times C$ such that

$$
\begin{cases}\left\langle\mu_{1} B_{1} y^{*}+x^{*}-y^{*}, x-x^{*}\right\rangle \geq 0, & \forall x \in C, \\ \left\langle\mu_{2} B_{2} x^{*}+y^{*}-x^{*}, x-y^{*}\right\rangle \geq 0, & \forall x \in C,\end{cases}
$$

which $\mu_{1}$ and $\mu_{2}$ are two positive constants. The set of solutions of problem (1.2) is still denoted by $\operatorname{GSVI}\left(C, B_{1}, B_{2}\right)$. In particular, if $B_{1}=B_{2}=A$, then problem (1.2) reduces to the new system of variational inequalities (NSVI), introduced and studied by Verma [4]. Further, if $x^{*}=y^{*}$ additionally, then the NSVI reduces to the classical variational inequality problem (VIP) of finding $x^{*} \in C$ such that

$$
\left\langle A x^{*}, x-x^{*}\right\rangle \geq 0, \quad \forall x \in C
$$

The solution set of $\operatorname{VIP}(1.3)$ is denoted by $\operatorname{VI}(C, A)$. Variational inequality theory has been studied quite extensively and has emerged as an important tool in the study of a wide class of obstacle, unilateral, free, moving, equilibrium problems. It is now well known that the variational inequalities are equivalent to the fixed point problems, the origin of which can be traced back to Lions and Stampacchia [5]. This alternative formulation has been used to suggest and analyze projection iterative method for solving variational inequalities under the conditions that the involved operator must be strongly monotone and Lipschitz continuous.

Recently, Ceng et al. [6] transformed problem (1.2) into a fixed point problem in the following way.

Lemma 1.1 (See [6]) For given $\bar{x}, \bar{y} \in C,(\bar{x}, \bar{y})$ is a solution of problem (1.2) if and only if $\bar{x}$ is a fixed point of the mapping $G: C \rightarrow C$ defined by

$$
G(x)=P_{C}\left[P_{C}\left(x-\mu_{2} B_{2} x\right)-\mu_{1} B_{1} P_{C}\left(x-\mu_{2} B_{2} x\right)\right], \quad \forall x \in C,
$$

where $\bar{y}=P_{C}\left(\bar{x}-\mu_{2} B_{2} \bar{x}\right)$ and $P_{C}$ is the projection of $H$ onto $C$.

In particular, if the mappings $B_{i}: C \rightarrow H$ is $\beta_{i}$-inverse strongly monotone for $i=1,2$, then the mapping $G$ is nonexpansive provided $\mu_{i} \in\left(0,2 \beta_{i}\right)$ for $i=1,2$.

In 1976, Korpelevich [7] proposed an iterative algorithm for solving the VIP (1.3) in Euclidean space $\mathbf{R}^{n}$ :

$$
\left\{\begin{array}{l}
y_{n}=P_{C}\left(x_{n}-\tau A x_{n}\right), \\
x_{n+1}=P_{C}\left(x_{n}-\tau A y_{n}\right), \quad n \geq 0,
\end{array}\right.
$$

with $\tau>0$ a given number, which is known as the extragradient method. The literature on the VIP is vast, and Korpelevich's extragradient method has received great attention given by many authors, who improved it in various ways; see, e.g., [3, 8-14] and the references therein, to name but a few. 
In particular, whenever $X$ is still a real smooth Banach space, $B_{1}=B_{2}=A$ and $x^{*}=y^{*}$, then GSVI (1.1) reduces to the variational inequality problem (VIP) of finding $x^{*} \in C$ such that

$$
\left\langle A x^{*}, J\left(x-x^{*}\right)\right\rangle \geq 0, \quad \forall x \in C,
$$

which was considered by Aoyama et al. [15]. Note that VIP (1.5) is connected with the fixed point problem for nonlinear mapping (see, e.g., $[16,17])$, the problem of finding a zero point of a nonlinear operator (see, e.g., [18]) and so on. It is clear that VIP (1.5) extends VIP (1.3) from Hilbert spaces to Banach spaces.

In order to find a solution of VIP (1.5), Aoyama et al. [15] introduced the following Mann iterative scheme for an accretive operator $A$ :

$$
x_{n+1}=\alpha_{n} x_{n}+\left(1-\alpha_{n}\right) \Pi_{C}\left(x_{n}-\lambda_{n} A x_{n}\right), \quad \forall n \geq 1,
$$

where $\Pi_{C}$ is a sunny nonexpansive retraction from $X$ onto $C$. Then they proved a weak convergence theorem.

Obviously, it is an interesting and valuable problem of constructing some algorithms with strong convergence for solving GSVI (1.1), which contains VIP (1.5) as a special case. Very recently, Cai and $\mathrm{Bu}$ [3] constructed an iterative algorithm for solving GSVI (1.1) and a common fixed point problem of a countable family of nonexpansive mappings in a uniformly convex and 2-uniformly smooth Banach space.

Theorem 1.2 (See [3, Theorem 3.1]) Let $C$ be a nonempty closed convex subset of a uniformly convex and 2-uniformly smooth Banach space $X$. Let $\Pi_{C}$ be a sunny nonexpansive retraction from $X$ onto $C$. Let the mapping $B_{i}: C \rightarrow X$ be $\beta_{i}$-inverse-strongly accretive with $0<\mu_{i}<\frac{\beta_{i}}{\kappa^{2}}$ for $i=1,2$. Let $f$ be a contraction of $C$ into itself with coeffcient $\delta \in(0,1)$. Let $\left\{T_{n}\right\}_{n=1}^{\infty}$ be a countable family of nonexpansive mappings of $C$ into itself such that $F=\bigcap_{i=1}^{\infty} \operatorname{Fix}\left(T_{i}\right) \cap \Omega \neq \emptyset$, where $\Omega$ is the fixed point set of the mapping $G=\Pi_{C}\left(I-\mu_{1} B_{1}\right) \Pi_{C}\left(I-\mu_{2} B_{2}\right)$ on $C$. For arbitrarily given $x_{1} \in C$, let $\left\{x_{n}\right\}$ be the sequence generated by

$$
\left\{\begin{array}{l}
x_{n+1}=\beta_{n} x_{n}+\left(1-\beta_{n}\right) T_{n} y_{n}, \\
y_{n}=\alpha_{n} f\left(x_{n}\right)+\left(1-\alpha_{n}\right) z_{n}, \\
z_{n}=\Pi_{C}\left(u_{n}-\mu_{1} B_{1} u_{n}\right), \\
u_{n}=\Pi_{C}\left(x_{n}-\mu_{2} B_{2} x_{n}\right), \quad \forall n \geq 1 .
\end{array}\right.
$$

Suppose that $\left\{\alpha_{n}\right\}$ and $\left\{\beta_{n}\right\}$ are two sequences in $(0,1)$ satisfying the following conditions:

(i) $\lim _{n \rightarrow \infty} \alpha_{n}=0$ and $\sum_{n=1}^{\infty} \alpha_{n}=\infty$;

(ii) $0<\liminf _{n \rightarrow \infty} \beta_{n} \leq \limsup _{n \rightarrow \infty} \beta_{n}<1$.

Assume that $\sum_{n=1}^{\infty} \sup _{x \in D}\left\|T_{n+1} x-T_{n} x\right\|<\infty$ for any bounded subset $D$ of $C$, and let $T$ be a mapping of $C$ into $X$ defined by $T x=\lim _{n \rightarrow \infty} T_{n} x$ for all $x \in C$ and suppose that $\operatorname{Fix}(T)=$ $\bigcap_{n=1}^{\infty} \operatorname{Fix}\left(T_{n}\right)$. Then $\left\{x_{n}\right\}$ converges strongly to $q \in F$, which solves the following VIP:

$$
\langle q-f(q), J(q-p)\rangle \leq 0, \quad \forall p \in F
$$


Furthermore, recall that a (possibly multivalued) operator $A \subset X \times X$ with domain $D(A)$ and range $R(A)$ in a real Banach space $X$ is accretive if, for each $x_{i} \in D(A)$ and $y_{i} \in A x_{i}$ $(i=1,2)$, there exists a $j\left(x_{1}-x_{2}\right) \in J\left(x_{1}-x_{2}\right)$ such that $\left\langle y_{1}-y_{2}, j\left(x_{1}-x_{2}\right)\right\rangle \geq 0$. (Here $J$ is the duality mapping.) An accretive operator $A$ is said to satisfy the range condition if $\overline{D(A)} \subset R(I+r A)$ for all $r>0$. An accretive operator $A$ is $m$-accretive if $R(I+r A)=X$ for each $r>0$. If $A$ is an accretive operator which satisfies the range condition, then we can define, for each $r>0$ a mapping $J_{r}: R(I+r A) \rightarrow D(A)$ defined by $J_{r}=(I+r A)^{-1}$, which is called the resolvent of $A$. We know that $J_{r}$ is nonexpansive and Fix $\left(J_{r}\right)=A^{-1} 0$ for all $r>0$. Hence,

$$
\operatorname{Fix}\left(J_{r}\right)=A^{-1} 0=\{z \in D(A): 0 \in A z\} .
$$

If $A^{-1} 0 \neq \emptyset$, then the inclusion $0 \in A z$ is solvable. The following resolvent identity is well known to us; see [19], where more details on accretive operators can be found.

Proposition 1.1 (Resolvent identity) For $\lambda>0, \mu>0$ and $x \in X$,

$$
J_{\lambda} x=J_{\mu}\left(\frac{\mu}{\lambda} x+\left(1-\frac{\mu}{\lambda}\right) J_{\lambda} x\right)
$$

Recently, Aoyama et al. [20] studied the following iterative scheme in a uniformly convex Banach space having a uniformly Gâteaux differentiable norm: for resolvents $J_{r_{n}}$ of an accretive operator $A$ such that $A^{-1} 0 \neq \emptyset$ and $\overline{D(A)} \subset C \subset \bigcap_{r>0} R(I+r A)$ and $\left\{\alpha_{n}\right\} \subset(0,1)$

$$
\left\{\begin{array}{l}
x_{0}=x \in C, \\
x_{n+1}=\alpha_{n} x+\left(1-\alpha_{n}\right) J_{r_{n}} x_{n} .
\end{array}\right.
$$

They proved that the sequence $\left\{x_{n}\right\}$ generated by (1.7) converges strongly to a zero of $A$ under appropriate assumptions on $\left\{\alpha_{n}\right\}$ and $\left\{r_{n}\right\}$. Subsequently, Ceng et al. [21] introduced and analyzed the following composite iterative scheme in either a uniformly smooth $\mathrm{Ba}$ nach space or a reflexive Banach space having a weakly sequentially continuous duality mapping

$$
\left\{\begin{array}{l}
x_{0}=x \in X, \\
y_{n}=\alpha_{n} u+\left(1-\alpha_{n}\right) J_{r_{n}} x_{n}, \\
x_{n+1}=\left(1-\beta_{n}\right) y_{n}+\beta_{n} J_{r_{n}} y_{n},
\end{array}\right.
$$

where $u \in \overline{D(A)}$ is an arbitrary (but fixed) element, under the following control conditions:

(H1) $\lim _{n \rightarrow \infty} \alpha_{n}=0$;

(H2) $\sum_{n=0}^{\infty} \alpha_{n}=\infty$, or, equivalently, $\prod_{n=0}^{\infty}\left(1-\alpha_{n}\right)=0$;

(H3) $\sum_{n=1}^{\infty}\left|\alpha_{n}-\alpha_{n-1}\right|<\infty$;

(H4) $r_{n} \geq \varepsilon, \forall n \geq 0$, for some $\varepsilon>0$ and $\sum_{n=1}^{\infty}\left|r_{n}-r_{n-1}\right|<\infty$;

(H5) $\beta_{n} \in[0, a)$ for some $a \in(0,1)$ and $\sum_{n=1}^{\infty}\left|\beta_{n}-\beta_{n-1}\right|<\infty$.

Further, as the viscosity approximation method, Jung [22] purposed and analyzed the following composite iterative scheme for finding a zero of an accretive operator $A$ : for 
resolvent $J_{r_{n}}$ of an accretive operator $A$ such that $A^{-1} 0 \neq \emptyset$ and $\overline{D(A)} \subset C \subset \bigcap_{r>0} R(I+r A)$, $f \in \Xi_{C}\left(\Xi_{C}\right.$ denotes the set of all contractions on $C$ ) and $\left\{\alpha_{n}\right\},\left\{\beta_{n}\right\} \subset(0,1)$,

$$
\left\{\begin{array}{l}
x_{0}=x \in C, \\
y_{n}=\alpha_{n} f\left(x_{n}\right)+\left(1-\alpha_{n}\right) J_{r_{n}} x_{n}, \\
x_{n+1}=\left(1-\beta_{n}\right) y_{n}+\beta_{n} J_{r_{n}} y_{n} .
\end{array}\right.
$$

Theorem 1.3 (See [22, Theorem 3.1]) Let X be a strictly convex and reflexive Banach space having a uniformly Gâteaux differentiable norm. Let $C$ be a nonempty closed convex subset of $X$ and $A \subset X \times X$ an accretive operator in $X$ such that $A^{-1} 0 \neq \emptyset$ and $\overline{D(A)} \subset C \subset$ $\bigcap_{r>0} R(I+r A)$. Let $\left\{\alpha_{n}\right\}$ and $\left\{\beta_{n}\right\}$ be sequences in $(0,1)$ which satisfy the conditions:

(i) $\lim _{n \rightarrow \infty} \alpha_{n}=0$ and $\sum_{n=0}^{\infty} \alpha_{n}=\infty$;

(ii) $\beta_{n} \in[0, a)$ for some $0<a<1$ for all $n \geq 0$.

Let $f \in \Xi_{C}$ and $x_{0} \in C$ be chosen arbitrarily. Let $\left\{x_{n}\right\}$ be a sequence generated by (JS) for $r_{n}>0$. If $\left\{x_{n}\right\}$ is asymptotically regular, i.e., $\lim _{n \rightarrow \infty}\left\|x_{n+1}-x_{n}\right\|=0$, then $\left\{x_{n}\right\}$ converges strongly to $q \in A^{-1} 0$, which is the unique solution of the variational inequality problem $(V I P)$

$$
\langle(I-f) q, J(q-p)\rangle \leq 0, \quad \forall f \in \Xi_{C}, p \in A^{-1} 0 .
$$

Let $C$ be a nonempty closed convex subset of a real smooth Banach space $X$. Let $\Pi_{C}$ be a sunny nonexpansive retraction from $X$ onto $C$, and let $f: C \rightarrow C$ be a contraction with coefficient $\rho \in(0,1)$. Motivated and inspired by the research going on in this area, we introduce the composite Mann iteration methods for finding solutions of GSVI (1.1), which are also common fixed points of a countable family of nonexpansive mappings and zeros of an accretive operator $A \subset X \times X$ such that $\overline{D(A)} \subset C \subset \bigcap_{r>0} R(I+r A)$. Here, the composite Mann iteration methods are based on Korpelevich's extragradient method, viscosity approximation method and the Mann iteration method. We first consider and analyze a composite Mann iterative algorithm in the setting of uniformly convex and 2uniformly smooth Banach space, and then another composite Mann iterative algorithm in a uniformly convex Banach space having a uniformly Gâteaux differentiable norm. Under suitable assumptions, we derive some strong convergence theorems. The results presented in this paper improve, extend, supplement and develop the corresponding results announced in the earlier and very recent literature; see, e.g., $[2,3,6,8,22]$.

\section{Preliminaries}

Let $X$ be a real Banach space. We define a function $\rho:[0, \infty) \rightarrow[0, \infty)$ called the modulus of smoothness of $X$ as follows:

$$
\rho(\tau)=\sup \left\{\frac{1}{2}(\|x+y\|+\|x-y\|)-1: x, y \in X,\|x\|=1,\|y\|=\tau\right\} .
$$

It is known that $X$ is uniformly smooth if and only if $\lim _{\tau \rightarrow 0} \rho(\tau) / \tau=0$. Let $q$ be a fixed real number with $1<q \leq 2$. Then a Banach space $X$ is said to be $q$-uniformly smooth if there exists a constant $c>0$ such that $\rho(\tau) \leq c \tau^{q}$ for all $\tau>0$. As pointed out in [23], no Banach space is $q$-uniformly smooth for $q>2$. In addition, it is also known that $J$ is 
single-valued if and only if $X$ is smooth, whereas if $X$ is uniformly smooth, then $J$ is normto-norm uniformly continuous on bounded subsets of $X$. If $X$ has a uniformly Gâteaux differentiable norm, then the duality mapping $J$ is norm-to-weak* uniformly continuous on bounded subsets of $X$. We use the notation $\rightarrow$ to indicate the weak convergence and the one $\rightarrow$ to indicate the strong convergence.

Let $C$ be a nonempty closed convex subset of $X$. Recall that a mapping $A: C \rightarrow X$ is said to be

(i) $\alpha$-strongly accretive if for each $x, y \in C$, there exists $j(x-y) \in J(x-y)$ such that

$$
\langle A x-A y, j(x-y)\rangle \geq \alpha\|x-y\|^{2}
$$

for some $\alpha \in(0,1)$;

(ii) $\beta$-inverse-strongly-accretive if for each $x, y \in C$, there exists $j(x-y) \in J(x-y)$ such that

$$
\langle A x-A y, j(x-y)\rangle \geq \beta\|A x-A y\|^{2}
$$

for some $\beta>0$;

(iii) $\lambda$-strictly pseudocontractive [24] if for each $x, y \in C$, there exists $j(x-y) \in J(x-y)$ such that

$$
\langle A x-A y, j(x-y)\rangle \leq\|x-y\|^{2}-\lambda\|x-y-(A x-A y)\|^{2}
$$

for some $\lambda \in(0,1)$.

It is worth emphasizing that the definition of the inverse strongly accretive mapping is based on that of the inverse strongly monotone mapping, which was studied by so many authors; see, e.g., $[9,25,26]$.

Proposition 2.1 (See [27]) Let X be a 2-uniformly smooth Banach space. Then

$$
\|x+y\|^{2} \leq\|x\|^{2}+2\langle y, J(x)\rangle+2\|\kappa y\|^{2}, \quad \forall x, y \in X,
$$

where $\kappa$ is the 2-uniformly smooth constant of $X$, and $J$ is the normalized duality mapping from $X$ into $X^{*}$.

Proposition 2.2 (See [28]) Let $X$ be a real smooth and uniform convex Banach space, and let $r>0$. Then there exists a strictly increasing, continuous and convex function $g:[0,2 r] \rightarrow \mathbf{R}, g(0)=0$ such that

$$
g(\|x-y\|) \leq\|x\|^{2}-2\langle x, J(y)\rangle+\|y\|^{2}, \quad \forall x, y \in B_{r}
$$

where $B_{r}=\{x \in X:\|x\| \leq r\}$.

Next, we list some lemmas that will be used in the sequel. Lemma 2.1 can be found in [29]. Lemma 2.2 is an immediate consequence of the subdifferential inequality of the function $\frac{1}{2}\|\cdot\|^{2}$. 
Lemma 2.1 Let $\left\{s_{n}\right\}$ be a sequence of nonnegative real numbers satisfying

$$
s_{n+1} \leq\left(1-\alpha_{n}\right) s_{n}+\alpha_{n} \beta_{n}+\gamma_{n}, \quad \forall n \geq 0,
$$

where $\left\{\alpha_{n}\right\},\left\{\beta_{n}\right\}$ and $\left\{\gamma_{n}\right\}$ satisfy the conditions

(i) $\left\{\alpha_{n}\right\} \subset[0,1]$ and $\sum_{n=0}^{\infty} \alpha_{n}=\infty$;

(ii) $\lim \sup _{n \rightarrow \infty} \beta_{n} \leq 0$;

(iii) $\gamma_{n} \geq 0, \forall n \geq 0$, and $\sum_{n=0}^{\infty} \gamma_{n}<\infty$

Then $\lim \sup _{n \rightarrow \infty} s_{n}=0$.

Lemma 2.2 In a real smooth Banach space X, the following inequality holds:

$$
\|x+y\|^{2} \leq\|x\|^{2}+2\langle y, J(x+y)\rangle, \quad \forall x, y \in X
$$

Let $D$ be a subset of $C$, and let $\Pi$ be a mapping of $C$ into $D$. Then $\Pi$ is said to be sunny if

$$
\Pi[\Pi(x)+t(x-\Pi(x))]=\Pi(x)
$$

whenever $\Pi(x)+t(x-\Pi(x)) \in C$ for $x \in C$ and $t \geq 0$. A mapping $\Pi$ of $C$ into itself is called a retraction if $\Pi^{2}=\Pi$. If a mapping $\Pi$ of $C$ into itself is a retraction, then $\Pi(z)=z$ for every $z \in R(\Pi)$, where $R(\Pi)$ is the range of $\Pi$. A subset $D$ of $C$ is called a sunny nonexpansive retract of $C$ if there exists a sunny nonexpansive retraction from $C$ onto $D$. The following lemma concerns the sunny nonexpansive retraction.

Lemma 2.3 (See [30]) Let C be a nonempty closed convex subset of a real smooth Banach space $X$. Let $D$ be a nonempty subset of $C$. Let $\Pi$ be a retraction of $C$ onto $D$. Then the following are equivalent:

(i) $\Pi$ is sunny and nonexpansive;

(ii) $\|\Pi(x)-\Pi(y)\|^{2} \leq\langle x-y, J(\Pi(x)-\Pi(y))\rangle, \forall x, y \in C$;

(iii) $\langle x-\Pi(x), J(y-\Pi(x))\rangle \leq 0, \forall x \in C, y \in D$.

It is well known that if $X=H$ in a Hilbert space, then a sunny nonexpansive retraction $\Pi_{C}$ is coincident with the metric projection from $X$ onto $C$; that is, $\Pi_{C}=P_{C}$. If $C$ is a nonempty closed convex subset of a strictly convex and uniformly smooth Banach space $X$, and if $T: C \rightarrow C$ is a nonexpansive mapping with the fixed point set $\operatorname{Fix}(T) \neq \emptyset$, then the set $\operatorname{Fix}(T)$ is a sunny nonexpansive retract of $C$.

Lemma 2.4 Let $C$ be a nonempty closed convex subset of a smooth Banach space $X$. Let $\Pi_{C}$ be a sunny nonexpansive retraction from $X$ onto $C$, and let $B_{1}, B_{2}: C \rightarrow X$ be nonlinear mappings. For given $x^{*}, y^{*} \in C,\left(x^{*}, y^{*}\right)$ is a solution of GSVI (1.1) if and only if $x^{*}=\Pi_{C}\left(y^{*}-\right.$ $\left.\mu_{1} B_{1} y^{*}\right)$, where $y^{*}=\Pi_{C}\left(x^{*}-\mu_{2} B_{2} x^{*}\right)$.

Proof We can rewrite GSVI (1.1) as

$$
\begin{cases}\left\langle x^{*}-\left(y^{*}-\mu_{1} B_{1} y^{*}\right), J\left(x-x^{*}\right)\right\rangle \geq 0, & \forall x \in C, \\ \left\langle y^{*}-\left(x^{*}-\mu_{2} B_{2} x^{*}\right), J\left(x-y^{*}\right)\right\rangle \geq 0, & \forall x \in C,\end{cases}
$$


which is obviously equivalent to

$$
\left\{\begin{array}{l}
x^{*}=\Pi_{C}\left(y^{*}-\mu_{1} B_{1} y^{*}\right), \\
y^{*}=\Pi_{C}\left(x^{*}-\mu_{2} B_{2} x^{*}\right),
\end{array}\right.
$$

because of Lemma 2.3. This completes the proof.

In terms of Lemma 2.4, we observe that

$$
x^{*}=\Pi_{C}\left[\Pi_{C}\left(x^{*}-\mu_{2} B_{2} x^{*}\right)-\mu_{1} B_{1} \Pi_{C}\left(x^{*}-\mu_{2} B_{2} x^{*}\right)\right],
$$

which implies that $x^{*}$ is a fixed point of the mapping $G$. Throughout this paper, the set of fixed points of the mapping $G$ is denoted by $\Omega$.

Lemma 2.5 (See [27]) Given a number $r>0$. A real Banach space $X$ is uniformly convex if and only if there exists a continuous strictly increasing function $g:[0, \infty) \rightarrow[0, \infty)$, $g(0)=0$ such that

$$
\|\lambda x+(1-\lambda) y\|^{2} \leq \lambda\|x\|^{2}+(1-\lambda)\|y\|^{2}-\lambda(1-\lambda) g(\|x-y\|)
$$

for all $\lambda \in[0,1]$ and $x, y \in X$ such that $\|x\| \leq r$ and $\|y\| \leq r$.

Lemma 2.6 (See [31]) Let C be a nonempty closed convex subset of a Banach space X. Let $S_{0}, S_{1}, \ldots$ be a sequence of mappings of $C$ into itself. Suppose that $\sum_{n=1}^{\infty} \sup \left\{\left\|S_{n} x-S_{n-1} x\right\|\right.$ : $x \in C\}<\infty$. Then for each $y \in C,\left\{S_{n} y\right\}$ converges strongly to some point of $C$. Moreover, let $S$ be a mapping of $C$ into itself defined by $S y=\lim _{n \rightarrow \infty} S_{n} y$ for all $y \in C$. Then $\lim _{n \rightarrow \infty} \sup \left\{\left\|S x-S_{n} x\right\|: x \in C\right\}=0$.

Let $C$ be a nonempty closed convex subset of a Banach space $X$, and let $T: C \rightarrow C$ be a nonexpansive mapping with $\operatorname{Fix}(T) \neq \emptyset$. As previously, let $\Xi_{C}$ be the set of all contractions on $C$. For $t \in(0,1)$ and $f \in \Xi_{C}$, let $x_{t} \in C$ be the unique fixed point of the contraction $x \mapsto t f(x)+(1-t) T x$ on $C$; that is,

$$
x_{t}=t f\left(x_{t}\right)+(1-t) T x_{t} .
$$

Lemma 2.7 (See $[17,32])$ Let $X$ be a uniformly smooth Banach space, or a reflexive and strictly convex Banach space with a uniformly Gâteaux differentiable norm. Let $C$ be a nonempty closed convex subset of $X$, let $T: C \rightarrow C$ be a nonexpansive mapping with $\operatorname{Fix}(T) \neq \emptyset$, and $f \in \Xi_{C}$. Then the net $\left\{x_{t}\right\}$ defined by $x_{t}=t f\left(x_{t}\right)+(1-t) T x_{t}$ converges strongly to a point in $\operatorname{Fix}(T)$. If we define a mapping $Q: \Xi_{C} \rightarrow \operatorname{Fix}(T)$ by $Q(f):=s-\lim _{t \rightarrow 0} x_{t}$, $\forall f \in \Xi_{C}$, then $Q(f)$ solves the VIP:

$$
\langle(I-f) Q(f), J(Q(f)-p)\rangle \leq 0, \quad \forall f \in \Xi_{C}, p \in \operatorname{Fix}(T) .
$$

Lemma 2.8 (See [33]) Let $C$ be a nonempty closed convex subset of a strictly convex Banach space $X$. Let $\left\{T_{n}\right\}_{n=0}^{\infty}$ be a sequence of nonexpansive mappings on C. Suppose that 
$\bigcap_{n=0}^{\infty} \operatorname{Fix}\left(T_{n}\right)$ is nonempty. Let $\left\{\lambda_{n}\right\}$ be a sequence of positive numbers with $\sum_{n=0}^{\infty} \lambda_{n}=1$. Then a mapping $S$ on $C$ defined by $S x=\sum_{n=0}^{\infty} \lambda_{n} T_{n} x$ for $x \in C$ is defined well, nonexpansive and $\operatorname{Fix}(S)=\bigcap_{n=0}^{\infty} \operatorname{Fix}\left(T_{n}\right)$ holds.

Lemma 2.9 (See [15]) Let $C$ be a nonempty closed convex subset of a smooth Banach space $X$. Let $\Pi_{C}$ be a sunny nonexpansive retraction from $X$ onto $C$, and let $A: C \rightarrow X$ be an accretive mapping. Then for all $\lambda>0$,

$$
\operatorname{VI}(C, A)=\operatorname{Fix}\left(\Pi_{C}(I-\lambda A)\right)
$$

Lemma 2.10 (See [34]) Let $\left\{x_{n}\right\}$ and $\left\{z_{n}\right\}$ be bounded sequences in a Banach space $X$, and let $\left\{\beta_{n}\right\}$ be a sequence of nonnegative numbers in $[0,1]$ with $0<\liminf _{n \rightarrow \infty} \beta_{n} \leq$ $\limsup _{n \rightarrow \infty} \beta_{n}<1$. Suppose that $x_{n+1}=\beta_{n} x_{n}+\left(1-\beta_{n}\right) z_{n}$ for all integers $n \geq 0$ and $\lim \sup _{n \rightarrow \infty}\left(\left\|z_{n+1}-z_{n}\right\|-\left\|x_{n+1}-x_{n}\right\|\right) \leq 0$. Then $\lim _{n \rightarrow \infty}\left\|x_{n}-z_{n}\right\|=0$.

Lemma 2.11 (See [35]) Let $X$ be a uniformly convex Banach space and $B_{r}=\{x \in X:\|x\| \leq$ $r\}, r>0$. Then there exists a continuous, strictly increasing and convex function $g:[0, \infty] \rightarrow$ $[0, \infty], g(0)=0$ such that

$$
\|\alpha x+\beta y+\gamma z\|^{2} \leq \alpha\|x\|^{2}+\beta\|y\|^{2}+\gamma\|z\|^{2}-\alpha \beta g(\|x-y\|)
$$

for all $x, y, z \in B_{r}$ and all $\alpha, \beta, \gamma \in[0,1]$ with $\alpha+\beta+\gamma=1$.

\section{Composite Mann iterative algorithms in uniformly convex and 2-uniformly smooth Banach spaces}

In this section, we introduce our composite Mann iterative algorithms in uniformly convex and 2-uniformly smooth Banach spaces and show the strong convergence theorems. We will use some useful lemmas in the sequel.

Lemma 3.1 (See [3, Lemma 2.8]) Let $C$ be a nonempty closed convex subset of a real 2uniformly smooth Banach space $X$. Let the mapping $B_{i}: C \rightarrow X$ be $\alpha_{i}$-inverse-strongly accretive. Then we have

$$
\left\|\left(I-\mu_{i} B_{i}\right) x-\left(I-\mu_{i} B_{i}\right) y\right\|^{2} \leq\|x-y\|^{2}+2 \mu_{i}\left(\mu_{i} \kappa^{2}-\alpha_{i}\right)\left\|B_{i} x-B_{i} y\right\|^{2}, \quad \forall x, y \in C,
$$

for $i=1,2$, where $\mu_{i}>0$. In particular, if $0<\mu_{i} \leq \frac{\alpha_{i}}{\kappa^{2}}$, then $I-\mu_{i} B_{i}$ is nonexpansive for $i=1,2$.

Lemma 3.2 (See [3, Lemma 2.9]) Let $C$ be a nonempty closed convex subset of a real 2 -uniformly smooth Banach space $X$. Let $\Pi_{C}$ be a sunny nonexpansive retraction from $X$ onto $C$. Let the mapping $B_{i}: C \rightarrow X$ be $\alpha_{i}$-inverse-strongly accretive for $i=1,2$. Let $G: C \rightarrow C$ be the mapping defined by

$$
G x=\Pi_{C}\left[\Pi_{C}\left(x-\mu_{2} B_{2} x\right)-\mu_{1} B_{1} \Pi_{C}\left(x-\mu_{2} B_{2} x\right)\right], \quad \forall x \in C .
$$

If $0<\mu_{i} \leq \frac{\alpha_{i}}{\kappa^{2}}$ for $i=1,2$, then $G: C \rightarrow C$ is nonexpansive. 
Theorem 3.1 Let $C$ be a nonempty closed convex subset of a uniformly convex and 2-uniformly smooth Banach space $X$. Let $\Pi_{C}$ be a sunny nonexpansive retraction from $X$ onto C. Let $A \subset X \times X$ be an accretive operator in $X$ such that $\overline{D(A)} \subset C \subset \bigcap_{r>0} R(I+r A)$. Let $B_{i}: C \rightarrow X$ be $\alpha_{i}$-inverse strongly accretive for $i=1,2$. Let $f: C \rightarrow C$ be a contraction with coefficient $\rho \in(0,1)$. Let $\left\{S_{i}\right\}_{i=0}^{\infty}$ be a countable family of nonexpansive mappings of $C$ into itself such that $F=\bigcap_{i=0}^{\infty} \operatorname{Fix}\left(S_{i}\right) \cap \Omega \cap A^{-1} 0 \neq \emptyset$, where $\Omega$ is the fixed point set of the mapping $G=\Pi_{C}\left(I-\mu_{1} B_{1}\right) \Pi_{C}\left(I-\mu_{2} B_{2}\right)$ with $0<\mu_{i}<\frac{\alpha_{i}}{\kappa^{2}}$ for $i=1$, 2. For arbitrarily given $x_{0} \in C$, let $\left\{x_{n}\right\}$ be the sequence generated by

$$
\left\{\begin{array}{l}
y_{n}=\beta_{n} x_{n}+\gamma_{n} S_{n} x_{n}+\delta_{n} J_{r_{n}} G x_{n}, \\
x_{n+1}=\alpha_{n} f\left(x_{n}\right)+\left(1-\alpha_{n}\right) y_{n}, \quad \forall n \geq 0,
\end{array}\right.
$$

where $\left\{\alpha_{n}\right\},\left\{\beta_{n}\right\},\left\{\gamma_{n}\right\}$ and $\left\{\delta_{n}\right\}$ are the sequences in $[0,1]$ such that $\beta_{n}+\gamma_{n}+\delta_{n}=1$ for all $n \geq 0$. Suppose that the following conditions hold:

(i) $\sum_{n=0}^{\infty} \alpha_{n}=\infty$ and $0 \leq \alpha_{n} \leq 1-\rho, \forall n \geq n_{0}$ for some integer $n_{0} \geq 0$;

(ii) $\liminf _{n \rightarrow \infty} \gamma_{n}>0$ and $\liminf _{n \rightarrow \infty} \delta_{n}>0$;

(iii) $\lim _{n \rightarrow \infty}\left(\left|\frac{\alpha_{n+1}}{1-\left(1-\alpha_{n+1}\right) \beta_{n+1}}-\frac{\alpha_{n}}{1-\left(1-\alpha_{n}\right) \beta_{n}}\right|+\left|\frac{\delta_{n+1}}{1-\beta_{n+1}}-\frac{\delta_{n}}{1-\beta_{n}}\right|\right)=0$;

(iv) $\lim _{n \rightarrow \infty}\left|r_{n+1}-r_{n}\right|=0$ and $r_{n} \geq \varepsilon>0$ for all $n \geq 0$;

(v) $0<\liminf _{n \rightarrow \infty} \beta_{n} \leq \limsup _{n \rightarrow \infty} \beta_{n}<1$.

Assume that $\sum_{n=0}^{\infty} \sup _{x \in D}\left\|S_{n+1} x-S_{n} x\right\|<\infty$ for any bounded subset $D$ of $C$, and let $S$ be a mapping of $C$ into itself defined by $S x=\lim _{n \rightarrow \infty} S_{n} x$ for all $x \in C$, and suppose that $\operatorname{Fix}(S)=$ $\bigcap_{i=0}^{\infty} \operatorname{Fix}\left(S_{i}\right)$. Then,

$$
x_{n} \rightarrow q \Longleftrightarrow \alpha_{n}\left(f\left(x_{n}\right)-x_{n}\right) \rightarrow 0,
$$

where $q \in F$ solves the following VIP:

$$
\langle q-f(q), J(q-p)\rangle \leq 0, \quad \forall p \in F .
$$

Proof First of all, let us show that the sequence $\left\{x_{n}\right\}$ is bounded. Indeed, take a fixed $p \in F$ arbitrarily. Then we get $p=G p, p=S_{n} p$ and $p=J_{r_{n}} p$ for all $n \geq 0$. By Lemma 3.2, we know that $G$ is nonexpansive. Then from (3.1), we have

$$
\begin{aligned}
\left\|y_{n}-p\right\| & \leq \beta_{n}\left\|x_{n}-p\right\|+\gamma_{n}\left\|S_{n} x_{n}-p\right\|+\delta_{n}\left\|J_{r_{n}} G x_{n}-p\right\| \\
& \leq \beta_{n}\left\|x_{n}-p\right\|+\gamma_{n}\left\|x_{n}-p\right\|+\delta_{n}\left\|G x_{n}-p\right\| \\
& \leq \beta_{n}\left\|x_{n}-p\right\|+\gamma_{n}\left\|x_{n}-p\right\|+\delta_{n}\left\|x_{n}-p\right\| \\
& =\left\|x_{n}-p\right\|,
\end{aligned}
$$

and hence

$$
\begin{aligned}
\left\|x_{n+1}-p\right\| & \leq \alpha_{n}\left\|f\left(x_{n}\right)-p\right\|+\left(1-\alpha_{n}\right)\left\|y_{n}-p\right\| \\
& \leq \alpha_{n}\left(\left\|f\left(x_{n}\right)-f(p)\right\|+\|f(p)-p\|\right)+\left(1-\alpha_{n}\right)\left\|y_{n}-p\right\| \\
& \leq \alpha_{n}\left(\rho\left\|x_{n}-p\right\|+\|f(p)-p\|\right)+\left(1-\alpha_{n}\right)\left\|x_{n}-p\right\|
\end{aligned}
$$




$$
\begin{aligned}
& =\left(1-\alpha_{n}(1-\rho)\right)\left\|x_{n}-p\right\|+\alpha_{n}(1-\rho) \frac{\|f(p)-p\|}{1-\rho} \\
& \leq \max \left\{\left\|x_{n}-p\right\|, \frac{\|f(p)-p\|}{1-\rho}\right\} .
\end{aligned}
$$

By induction, we obtain

$$
\left\|x_{n}-p\right\| \leq \max \left\{\left\|x_{0}-p\right\|, \frac{\|f(p)-p\|}{1-\rho}\right\}, \quad \forall n \geq 0
$$

Thus, $\left\{x_{n}\right\}$ is bounded, and so are the sequences $\left\{y_{n}\right\},\left\{G x_{n}\right\}$ and $\left\{f\left(x_{n}\right)\right\}$.

Let us show that

$$
\lim _{n \rightarrow \infty}\left\|x_{n+1}-x_{n}\right\|=0
$$

As a matter of fact, put $\sigma_{n}=\left(1-\alpha_{n}\right) \beta_{n}, \forall n \geq 0$. Then it follows from (i) and (v) that

$$
\beta_{n} \geq \sigma_{n}=\left(1-\alpha_{n}\right) \beta_{n} \geq(1-(1-\rho)) \beta_{n}=\rho \beta_{n}, \quad \forall n \geq n_{0},
$$

and hence

$$
0<\liminf _{n \rightarrow \infty} \sigma_{n} \leq \liminf _{n \rightarrow \infty} \sigma_{n}<1
$$

Define

$$
x_{n+1}=\sigma_{n} x_{n}+\left(1-\sigma_{n}\right) z_{n} .
$$

Observe that

$$
\begin{aligned}
z_{n+1}- & z_{n} \\
= & \frac{x_{n+2}-\sigma_{n+1} x_{n+1}}{1-\sigma_{n+1}}-\frac{x_{n+1}-\sigma_{n} x_{n}}{1-\sigma_{n}} \\
= & \frac{\alpha_{n+1} f\left(x_{n+1}\right)+\left(1-\alpha_{n+1}\right) y_{n+1}-\sigma_{n+1} x_{n+1}}{1-\sigma_{n+1}}-\frac{\alpha_{n} f\left(x_{n}\right)+\left(1-\alpha_{n}\right) y_{n}-\sigma_{n} x_{n}}{1-\sigma_{n}} \\
= & \left(\frac{\alpha_{n+1} f\left(x_{n+1}\right)}{1-\sigma_{n+1}}-\frac{\alpha_{n} f\left(x_{n}\right)}{1-\sigma_{n}}\right)-\frac{\left(1-\alpha_{n}\right)\left[\beta_{n} x_{n}+\gamma_{n} S_{n} x_{n}+\delta_{n} J_{r_{n}} G x_{n}\right]-\sigma_{n} x_{n}}{1-\sigma_{n}} \\
& +\frac{\left(1-\alpha_{n+1}\right)\left[\beta_{n+1} x_{n+1}+\gamma_{n+1} S_{n+1} x_{n+1}+\delta_{n+1} J_{r_{n+1}} G x_{n+1}\right]-\sigma_{n+1} x_{n+1}}{1-\sigma_{n+1}} \\
= & \left(\frac{\alpha_{n+1} f\left(x_{n+1}\right)}{1-\sigma_{n+1}}-\frac{\alpha_{n} f\left(x_{n}\right)}{1-\sigma_{n}}\right)+\frac{1-\alpha_{n+1}}{1-\sigma_{n+1}}\left(\gamma_{n+1} S_{n+1} x_{n+1}+\delta_{n+1} J_{r_{n+1}} G x_{n+1}\right) \\
& \left.-\frac{1-\alpha_{n}\left(\gamma_{n} S_{n} x_{n}+\delta_{n} J_{r_{n}} G x_{n}\right)}{1-\sigma_{n}}\right] \\
= & \left(\frac{\alpha_{n+1} f\left(x_{n+1}\right)}{1-\sigma_{n+1}}-\frac{\alpha_{n} f\left(x_{n}\right)}{1-\sigma_{n}}\right) \\
& +\frac{\left(1-\alpha_{n+1}\right)\left(1-\beta_{n+1}\right)}{1-\sigma_{n+1}}\left[\frac{\gamma_{n+1} S_{n+1} x_{n+1}+\delta_{n+1} J_{r_{n+1}} G x_{n+1}}{1-\beta_{n+1}}-\frac{\gamma_{n} S_{n} x_{n}+\delta_{n} J_{r_{n}} G x_{n}}{1-\beta_{n}}\right]
\end{aligned}
$$


Cent and Wen Fixed Point Theory and Applications 2013, 2013:249

Page 13 of 37

http://www.fixedpointtheoryandapplications.com/content/2013/1/249

$$
\begin{aligned}
& +\left[\frac{\left(1-\alpha_{n+1}\right)\left(1-\beta_{n+1}\right)}{1-\sigma_{n+1}}-\frac{\left(1-\alpha_{n}\right)\left(1-\beta_{n}\right)}{1-\sigma_{n}}\right] \frac{\gamma_{n} S_{n} x_{n}+\delta_{n} J_{r_{n}} G x_{n}}{1-\beta_{n}} \\
= & \frac{\alpha_{n+1}}{1-\sigma_{n+1}}\left(f\left(x_{n+1}\right)-f\left(x_{n}\right)\right)+\left(\frac{\alpha_{n+1}}{1-\sigma_{n+1}}-\frac{\alpha_{n}}{1-\sigma_{n}}\right) f\left(x_{n}\right) \\
& +\frac{\left(1-\alpha_{n+1}\right)\left(1-\beta_{n+1}\right)}{1-\sigma_{n+1}}\left[\frac{\gamma_{n+1}}{\gamma_{n+1}+\delta_{n+1}}\left(S_{n+1} x_{n+1}-S_{n} x_{n}\right)\right. \\
& +\left(\frac{\gamma_{n+1}}{\gamma_{n+1}+\delta_{n+1}}-\frac{\gamma_{n}}{\gamma_{n}+\delta_{n}}\right) S_{n} x_{n} \\
& \left.+\frac{\delta_{n+1}}{\gamma_{n+1}+\delta_{n+1}}\left(J_{r_{n+1}} G x_{n+1}-J_{r_{n}} G x_{n}\right)+\left(\frac{\delta_{n+1}}{\gamma_{n+1}+\delta_{n+1}}-\frac{\delta_{n}}{\gamma_{n}+\delta_{n}}\right) J_{r_{n}} G x_{n}\right] \\
& -\left(\frac{\alpha_{n+1}}{1-\sigma_{n+1}}-\frac{\alpha_{n}}{1-\sigma_{n}}\right) \frac{\gamma_{n} S_{n} x_{n}+\delta_{n} J_{r_{n}} G x_{n}}{\gamma_{n}+\delta_{n}} \\
= & \frac{\alpha_{n+1}}{1-\sigma_{n+1}}\left(f\left(x_{n+1}\right)-f\left(x_{n}\right)\right)+\left(\frac{\alpha_{n+1}}{1-\sigma_{n+1}}-\frac{\alpha_{n}}{1-\sigma_{n}}\right)\left(f\left(x_{n}\right)-\frac{\gamma_{n} S_{n} x_{n}+\delta_{n} J_{r_{n}} G x_{n}}{\gamma_{n}+\delta_{n}}\right) \\
& +\frac{1-\sigma_{n+1}-\alpha_{n+1}}{1-\sigma_{n+1}}\left[\frac{\gamma_{n+1}}{\gamma_{n+1}+\delta_{n+1}}\left(S_{n+1} x_{n+1}-S_{n} x_{n}\right)+\left(\frac{\gamma_{n+1}}{\gamma_{n+1}+\delta_{n+1}}-\frac{\gamma_{n}}{\gamma_{n}+\delta_{n}}\right) S_{n} x_{n}\right. \\
& \left.+\frac{\delta_{n+1}}{\gamma_{n+1}+\delta_{n+1}}\left(J_{r_{n+1}} G x_{n+1}-J_{r_{n}} G x_{n}\right)+\left(\frac{\delta_{n+1}}{\gamma_{n+1}+\delta_{n+1}}-\frac{\delta_{n}}{\gamma_{n}+\delta_{n}}\right) J_{r_{n}} G x_{n}\right]
\end{aligned}
$$

and hence

$$
\begin{aligned}
\| z_{n+1}- & z_{n} \| \\
\leq & \frac{\alpha_{n+1}}{1-\sigma_{n+1}}\left\|f\left(x_{n+1}\right)-f\left(x_{n}\right)\right\|+\left|\frac{\alpha_{n+1}}{1-\sigma_{n+1}}-\frac{\alpha_{n}}{1-\sigma_{n}}\right|\left\|f\left(x_{n}\right)-\frac{\gamma_{n} S_{n} x_{n}+\delta_{n} J_{r_{n}} G x_{n}}{\gamma_{n}+\delta_{n}}\right\| \\
& +\frac{1-\sigma_{n+1}-\alpha_{n+1}}{1-\sigma_{n+1}} \| \frac{\gamma_{n+1}}{\gamma_{n+1}+\delta_{n+1}}\left(S_{n+1} x_{n+1}-S_{n} x_{n}\right)+\left(\frac{\gamma_{n+1}}{\gamma_{n+1}+\delta_{n+1}}-\frac{\gamma_{n}}{\gamma_{n}+\delta_{n}}\right) S_{n} x_{n} \\
& +\frac{\delta_{n+1}}{\gamma_{n+1}+\delta_{n+1}}\left(J_{r_{n+1}} G x_{n+1}-J_{r_{n}} G x_{n}\right)+\left(\frac{\delta_{n+1}}{\gamma_{n+1}+\delta_{n+1}}-\frac{\delta_{n}}{\gamma_{n}+\delta_{n}}\right) J_{r_{n}} G x_{n} \| \\
\leq & \frac{\rho \alpha_{n+1}}{1-\sigma_{n+1}}\left\|x_{n+1}-x_{n}\right\|+\left|\frac{\alpha_{n+1}}{1-\sigma_{n+1}}-\frac{\alpha_{n}}{1-\sigma_{n}}\right|\left(\left\|f\left(x_{n}\right)\right\|+\left\|S_{n} x_{n}\right\|+\left\|J_{r_{n}} G x_{n}\right\|\right) \\
& +\frac{1-\sigma_{n+1}-\alpha_{n+1}}{1-\sigma_{n+1}}\left[\frac{\gamma_{n+1}}{\gamma_{n+1}+\delta_{n+1}}\left\|S_{n+1} x_{n+1}-S_{n} x_{n}\right\|+\left|\frac{\gamma_{n+1}}{\gamma_{n+1}+\delta_{n+1}}-\frac{\gamma_{n}}{\gamma_{n}+\delta_{n}}\right|\left\|S_{n} x_{n}\right\|\right. \\
& \left.+\frac{\delta_{n+1}}{\gamma_{n+1}+\delta_{n+1}}\left\|J_{r_{n+1}} G x_{n+1}-J_{r_{n}} G x_{n}\right\|+\left|\frac{\delta_{n+1}}{\gamma_{n+1}+\delta_{n+1}}-\frac{\delta_{n}}{\gamma_{n}+\delta_{n}}\right|\left\|J_{r_{n}} G x_{n}\right\|\right] .
\end{aligned}
$$

On the other hand, if $r_{n} \leq r_{n+1}$, using the resolvent identity in Proposition 1.1,

$$
J_{r_{n+1}} G x_{n+1}=J_{r_{n}}\left(\frac{r_{n}}{r_{n+1}} G x_{n+1}+\left(1-\frac{r_{n}}{r_{n+1}}\right) J_{r_{n+1}} G x_{n+1}\right) \text {, }
$$

we get

$$
\begin{aligned}
\left\|J_{r_{n+1}} G x_{n+1}-J_{r_{n}} G x_{n}\right\| & =\left\|J_{r_{n}}\left(\frac{r_{n}}{r_{n+1}} G x_{n+1}+\left(1-\frac{r_{n}}{r_{n+1}}\right) J_{r_{n+1}} G x_{n+1}\right)-J_{r_{n}} G x_{n}\right\| \\
& \leq \frac{r_{n}}{r_{n+1}}\left\|G x_{n+1}-G x_{n}\right\|+\left(1-\frac{r_{n}}{r_{n+1}}\right)\left\|J_{r_{n+1}} G x_{n+1}-G x_{n}\right\|
\end{aligned}
$$




$$
\begin{aligned}
& \leq\left\|x_{n+1}-x_{n}\right\|+\frac{r_{n+1}-r_{n}}{r_{n+1}}\left\|J_{r_{n+1}} G x_{n+1}-G x_{n}\right\| \\
& \leq\left\|x_{n+1}-x_{n}\right\|+\frac{1}{\varepsilon}\left|r_{n+1}-r_{n}\right|\left\|J_{r_{n+1}} G x_{n+1}-G x_{n}\right\| .
\end{aligned}
$$

If $r_{n+1} \leq r_{n}$, we derive in the similar way

$$
\left\|J_{r_{n+1}} G x_{n+1}-J_{r_{n}} G x_{n}\right\| \leq\left\|x_{n}-x_{n+1}\right\|+\frac{1}{\varepsilon}\left|r_{n}-r_{n+1}\right|\left\|J_{r_{n}} G x_{n}-G x_{n+1}\right\| .
$$

Thus, combining the above cases, we obtain

$$
\left\|J_{r_{n+1}} G x_{n+1}-J_{r_{n}} G x_{n}\right\| \leq\left\|x_{n}-x_{n+1}\right\|+M_{0}\left|r_{n}-r_{n+1}\right|, \quad \forall n \geq 0,
$$

where $\sup _{n \geq 0}\left\{\frac{1}{\varepsilon}\left(\left\|J_{r_{n+1}} G x_{n+1}-G x_{n}\right\|+\left\|J_{r_{n}} G x_{n}-G x_{n+1}\right\|\right)\right\} \leq M_{0}$ for some $M_{0}>0$. Substituting (3.8) for (3.7), we have

$$
\begin{aligned}
& \left\|z_{n+1}-z_{n}\right\| \\
& \leq \frac{\rho \alpha_{n+1}}{1-\sigma_{n+1}}\left\|x_{n+1}-x_{n}\right\|+\left|\frac{\alpha_{n+1}}{1-\sigma_{n+1}}-\frac{\alpha_{n}}{1-\sigma_{n}}\right|\left(\left\|f\left(x_{n}\right)\right\|+\left\|S_{n} x_{n}\right\|+\left\|J_{r_{n}} G x_{n}\right\|\right) \\
& +\frac{1-\sigma_{n+1}-\alpha_{n+1}}{1-\sigma_{n+1}}\left[\frac{\gamma_{n+1}}{\gamma_{n+1}+\delta_{n+1}}\left(\left\|S_{n+1} x_{n+1}-S_{n+1} x_{n}\right\|+\left\|S_{n+1} x_{n}-S_{n} x_{n}\right\|\right)\right. \\
& +\left|\frac{\gamma_{n+1}}{\gamma_{n+1}+\delta_{n+1}}-\frac{\gamma_{n}}{\gamma_{n}+\delta_{n}}\right|\left\|S_{n} x_{n}\right\|+\frac{\delta_{n+1}}{\gamma_{n+1}+\delta_{n+1}}\left(\left\|x_{n}-x_{n+1}\right\|+M_{0}\left|r_{n}-r_{n+1}\right|\right) \\
& \left.+\left|\frac{\delta_{n+1}}{\gamma_{n+1}+\delta_{n+1}}-\frac{\delta_{n}}{\gamma_{n}+\delta_{n}}\right|\left\|J_{r_{n}} G x_{n}\right\|\right] \\
& \leq \frac{\rho \alpha_{n+1}}{1-\sigma_{n+1}}\left\|x_{n+1}-x_{n}\right\|+\left|\frac{\alpha_{n+1}}{1-\sigma_{n+1}}-\frac{\alpha_{n}}{1-\sigma_{n}}\right|\left(\left\|f\left(x_{n}\right)\right\|+\left\|S_{n} x_{n}\right\|+\left\|J_{r_{n}} G x_{n}\right\|\right) \\
& +\frac{1-\sigma_{n+1}-\alpha_{n+1}}{1-\sigma_{n+1}}\left[\frac{\gamma_{n+1}}{\gamma_{n+1}+\delta_{n+1}}\left(\left\|x_{n+1}-x_{n}\right\|+\left\|S_{n+1} x_{n}-S_{n} x_{n}\right\|\right)\right. \\
& +\frac{\delta_{n+1}}{\gamma_{n+1}+\delta_{n+1}}\left(\left\|x_{n}-x_{n+1}\right\|+M_{0}\left|r_{n}-r_{n+1}\right|\right) \\
& \left.+\left|\frac{\delta_{n+1}}{\gamma_{n+1}+\delta_{n+1}}-\frac{\delta_{n}}{\gamma_{n}+\delta_{n}}\right|\left(\left\|S_{n} x_{n}\right\|+\left\|J_{r_{n}} G x_{n}\right\|\right)\right] \\
& =\frac{1-\sigma_{n+1}-\alpha_{n+1}(1-\rho)}{1-\sigma_{n+1}}\left\|x_{n+1}-x_{n}\right\| \\
& +\left|\frac{\alpha_{n+1}}{1-\sigma_{n+1}}-\frac{\alpha_{n}}{1-\sigma_{n}}\right|\left(\left\|f\left(x_{n}\right)\right\|+\left\|S_{n} x_{n}\right\|+\left\|J_{r_{n}} G x_{n}\right\|\right) \\
& +\frac{1-\sigma_{n+1}-\alpha_{n+1}}{1-\sigma_{n+1}}\left[\frac{\gamma_{n+1}}{\gamma_{n+1}+\delta_{n+1}}\left\|S_{n+1} x_{n}-S_{n} x_{n}\right\|+\frac{\delta_{n+1}}{\gamma_{n+1}+\delta_{n+1}} M_{0}\left|r_{n}-r_{n+1}\right|\right. \\
& \left.+\left|\frac{\delta_{n+1}}{\gamma_{n+1}+\delta_{n+1}}-\frac{\delta_{n}}{\gamma_{n}+\delta_{n}}\right|\left(\left\|S_{n} x_{n}\right\|+\left\|J_{r_{n}} G x_{n}\right\|\right)\right] \\
& \leq\left\|x_{n+1}-x_{n}\right\|+\left|\frac{\alpha_{n+1}}{1-\sigma_{n+1}}-\frac{\alpha_{n}}{1-\sigma_{n}}\right| M+\left\|S_{n+1} x_{n}-S_{n} x_{n}\right\|+M\left|r_{n}-r_{n+1}\right| \\
& +\left|\frac{\delta_{n+1}}{\gamma_{n+1}+\delta_{n+1}}-\frac{\delta_{n}}{\gamma_{n}+\delta_{n}}\right| M
\end{aligned}
$$




$$
\begin{aligned}
= & \left\|x_{n+1}-x_{n}\right\|+M\left(\left|\frac{\alpha_{n+1}}{1-\sigma_{n+1}}-\frac{\alpha_{n}}{1-\sigma_{n}}\right|+\left|\frac{\delta_{n+1}}{\gamma_{n+1}+\delta_{n+1}}-\frac{\delta_{n}}{\gamma_{n}+\delta_{n}}\right|+\left|r_{n+1}-r_{n}\right|\right) \\
& +\left\|S_{n+1} x_{n}-S_{n} x_{n}\right\|,
\end{aligned}
$$

which hence yields

$$
\begin{aligned}
& \left\|z_{n+1}-z_{n}\right\|-\left\|x_{n+1}-x_{n}\right\| \\
& \leq M\left(\left|\frac{\alpha_{n+1}}{1-\sigma_{n+1}}-\frac{\alpha_{n}}{1-\sigma_{n}}\right|+\left|\frac{\delta_{n+1}}{\gamma_{n+1}+\delta_{n+1}}-\frac{\delta_{n}}{\gamma_{n}+\delta_{n}}\right|+\left|r_{n+1}-r_{n}\right|\right) \\
& \quad+\left\|S_{n+1} x_{n}-S_{n} x_{n}\right\|,
\end{aligned}
$$

where $\sup _{n \geq 0}\left\{\left\|f\left(x_{n}\right)\right\|+\left\|S_{n} x_{n}\right\|+\left\|J_{r_{n}} G x_{n}\right\|+M_{0}\right\} \leq M$ for some $M>0$. So, from (3.9), conditions (iii), (iv) and the assumption on $\left\{S_{n}\right\}$, it follows that

$$
\limsup _{n \rightarrow \infty}\left(\left\|z_{n+1}-z_{n}\right\|-\left\|x_{n+1}-x_{n}\right\|\right) \leq 0
$$

Consequently, by Lemma 2.10, we have

$$
\lim _{n \rightarrow \infty}\left\|z_{n}-x_{n}\right\|=0
$$

It follows from (3.5) and (3.6) that

$$
\lim _{n \rightarrow \infty}\left\|x_{n+1}-x_{n}\right\|=\lim _{n \rightarrow \infty}\left(1-\sigma_{n}\right)\left\|z_{n}-x_{n}\right\|=0 .
$$

From (3.1), we have

$$
x_{n+1}-x_{n}=\alpha_{n}\left(f\left(x_{n}\right)-x_{n}\right)+\left(1-\alpha_{n}\right)\left(y_{n}-x_{n}\right) \text {, }
$$

which hence implies that

$$
\begin{aligned}
\rho\left\|y_{n}-x_{n}\right\| & =(1-(1-\rho))\left\|y_{n}-x_{n}\right\| \leq\left(1-\alpha_{n}\right)\left\|y_{n}-x_{n}\right\| \\
& =\left\|x_{n+1}-x_{n}-\alpha_{n}\left(f\left(x_{n}\right)-x_{n}\right)\right\| \\
& \leq\left\|x_{n+1}-x_{n}\right\|+\left\|\alpha_{n}\left(f\left(x_{n}\right)-x_{n}\right)\right\| .
\end{aligned}
$$

Since $x_{n+1}-x_{n} \rightarrow 0$ and $\alpha_{n}\left(f\left(x_{n}\right)-x_{n}\right) \rightarrow 0$, we get

$$
\lim _{n \rightarrow \infty}\left\|y_{n}-x_{n}\right\|=0 \text {. }
$$

Next, we show that $\left\|x_{n}-G x_{n}\right\| \rightarrow 0$ as $n \rightarrow \infty$.

Indeed, for simplicity, put $q=\Pi_{C}\left(p-\mu_{2} B_{2} p\right), u_{n}=\Pi_{C}\left(x_{n}-\mu_{2} B_{2} x_{n}\right)$ and $v_{n}=\Pi_{C}\left(u_{n}-\right.$ $\left.\mu_{1} B_{1} u_{n}\right)$. Then $v_{n}=G x_{n}$ for all $n \geq 0$. From Lemma 3.1, we have

$$
\begin{aligned}
\left\|u_{n}-q\right\|^{2} & =\left\|\Pi_{C}\left(x_{n}-\mu_{2} B_{2} x_{n}\right)-\Pi_{C}\left(p-\mu_{2} B_{2} p\right)\right\|^{2} \leq\left\|x_{n}-p-\mu_{2}\left(B_{2} x_{n}-B_{2} p\right)\right\|^{2} \\
& \leq\left\|x_{n}-p\right\|^{2}-2 \mu_{2}\left(\alpha_{2}-\kappa^{2} \mu_{2}\right)\left\|B_{2} x_{n}-B_{2} p\right\|^{2}
\end{aligned}
$$


and

$$
\begin{aligned}
\left\|v_{n}-p\right\|^{2} & =\left\|\Pi_{C}\left(u_{n}-\mu_{1} B_{1} u_{n}\right)-\Pi_{C}\left(q-\mu_{1} B_{1} q\right)\right\|^{2} \\
& \leq\left\|u_{n}-q-\mu_{1}\left(B_{1} u_{n}-B_{1} q\right)\right\|^{2} \\
& \leq\left\|u_{n}-q\right\|^{2}-2 \mu_{1}\left(\alpha_{1}-\kappa^{2} \mu_{1}\right)\left\|B_{1} u_{n}-B_{1} q\right\|^{2} .
\end{aligned}
$$

Substituting (3.13) for (3.14), we obtain

$$
\begin{aligned}
\left\|v_{n}-p\right\|^{2} \leq & \left\|x_{n}-p\right\|^{2}-2 \mu_{2}\left(\alpha_{2}-\kappa^{2} \mu_{2}\right)\left\|B_{2} x_{n}-B_{2} p\right\|^{2} \\
& -2 \mu_{1}\left(\alpha_{1}-\kappa^{2} \mu_{1}\right)\left\|B_{1} u_{n}-B_{1} q\right\|^{2} .
\end{aligned}
$$

From (3.1) and (3.15), we have

$$
\begin{aligned}
\| y_{n}- & p \|^{2} \\
\leq & \beta_{n}\left\|x_{n}-p\right\|^{2}+\gamma_{n}\left\|S_{n} x_{n}-p\right\|^{2}+\delta_{n}\left\|J_{r_{n}} G x_{n}-p\right\|^{2} \\
\leq & \beta_{n}\left\|x_{n}-p\right\|^{2}+\gamma_{n}\left\|x_{n}-p\right\|^{2}+\delta_{n}\left\|v_{n}-p\right\|^{2} \\
\leq & \beta_{n}\left\|x_{n}-p\right\|^{2}+\gamma_{n}\left\|x_{n}-p\right\|^{2}+\delta_{n}\left[\left\|x_{n}-p\right\|^{2}\right. \\
& \left.-2 \mu_{2}\left(\alpha_{2}-\kappa^{2} \mu_{2}\right)\left\|B_{2} x_{n}-B_{2} p\right\|^{2}-2 \mu_{1}\left(\alpha_{1}-\kappa^{2} \mu_{1}\right)\left\|B_{1} u_{n}-B_{1} q\right\|^{2}\right] \\
= & \left\|x_{n}-p\right\|^{2}-2 \delta_{n}\left[\mu_{2}\left(\alpha_{2}-\kappa^{2} \mu_{2}\right)\left\|B_{2} x_{n}-B_{2} p\right\|^{2}\right. \\
& \left.+2 \mu_{1}\left(\alpha_{1}-\kappa^{2} \mu_{1}\right)\left\|B_{1} u_{n}-B_{1} q\right\|^{2}\right],
\end{aligned}
$$

which hence implies that

$$
\begin{aligned}
2 \delta_{n} & {\left[\mu_{2}\left(\alpha_{2}-\kappa^{2} \mu_{2}\right)\left\|B_{2} x_{n}-B_{2} p\right\|^{2}+\mu_{1}\left(\alpha_{1}-\kappa^{2} \mu_{1}\right)\left\|B_{1} u_{n}-B_{1} q\right\|^{2}\right] } \\
& \leq\left\|x_{n}-p\right\|^{2}-\left\|y_{n}-p\right\|^{2} \\
& \leq\left(\left\|x_{n}-p\right\|+\left\|y_{n}-p\right\|\right)\left\|x_{n}-y_{n}\right\| .
\end{aligned}
$$

Since $0<\mu_{i}<\frac{\alpha_{i}}{\kappa^{2}}$ for $i=1,2$, and $\left\{x_{n}\right\}$ is bounded, we obtain from (3.12), (3.17) and condition (ii) that

$$
\lim _{n \rightarrow \infty}\left\|B_{2} x_{n}-B_{2} p\right\|=0 \quad \text { and } \quad \lim _{n \rightarrow \infty}\left\|B_{1} u_{n}-B_{1} q\right\|=0 .
$$

Utilizing Proposition 2.2 and Lemma 2.3, we have

$$
\begin{aligned}
\left\|u_{n}-q\right\|^{2}= & \left\|\Pi_{C}\left(x_{n}-\mu_{2} B_{2} x_{n}\right)-\Pi_{C}\left(p-\mu_{2} B_{2} p\right)\right\|^{2} \\
\leq & \left\langle x_{n}-\mu_{2} B_{2} x_{n}-\left(p-\mu_{2} B_{2} p\right), J\left(u_{n}-q\right)\right\rangle \\
= & \left\langle x_{n}-p, J\left(u_{n}-q\right)\right\rangle+\mu_{2}\left\langle B_{2} p-B_{2} x_{n}, J\left(u_{n}-q\right)\right\rangle \\
\leq & \frac{1}{2}\left[\left\|x_{n}-p\right\|^{2}+\left\|u_{n}-q\right\|^{2}-g_{1}\left(\left\|x_{n}-u_{n}-(p-q)\right\|\right)\right] \\
& +\mu_{2}\left\|B_{2} p-B_{2} x_{n}\right\|\left\|u_{n}-q\right\|,
\end{aligned}
$$


which implies that

$$
\left\|u_{n}-q\right\|^{2} \leq\left\|x_{n}-p\right\|^{2}-g_{1}\left(\left\|x_{n}-u_{n}-(p-q)\right\|\right)+2 \mu_{2}\left\|B_{2} p-B_{2} x_{n}\right\|\left\|u_{n}-q\right\| .
$$

In the same way, we derive

$$
\begin{aligned}
\left\|v_{n}-p\right\|^{2}= & \left\|\Pi_{C}\left(u_{n}-\mu_{1} B_{1} u_{n}\right)-\Pi_{C}\left(q-\mu_{1} B_{1} q\right)\right\|^{2} \\
\leq & \left\langle u_{n}-\mu_{1} B_{1} u_{n}-\left(q-\mu_{1} B_{1} q\right), J\left(v_{n}-p\right)\right\rangle \\
= & \left\langle u_{n}-q, J\left(v_{n}-p\right)\right\rangle+\mu_{1}\left\langle B_{1} q-B_{1} u_{n}, J\left(v_{n}-p\right)\right\rangle \\
\leq & \frac{1}{2}\left[\left\|u_{n}-q\right\|^{2}+\left\|v_{n}-p\right\|^{2}-g_{2}\left(\left\|u_{n}-v_{n}+(p-q)\right\|\right)\right] \\
& +\mu_{1}\left\|B_{1} q-B_{1} u_{n}\right\|\left\|v_{n}-p\right\|,
\end{aligned}
$$

which implies that

$$
\left\|v_{n}-p\right\|^{2} \leq\left\|u_{n}-q\right\|^{2}-g_{2}\left(\left\|u_{n}-v_{n}+(p-q)\right\|\right)+2 \mu_{1}\left\|B_{1} q-B_{1} u_{n}\right\|\left\|v_{n}-p\right\| .
$$

Substituting (3.19) for (3.20), we get

$$
\begin{gathered}
\left\|v_{n}-p\right\|^{2} \leq\left\|x_{n}-p\right\|^{2}-g_{1}\left(\left\|x_{n}-u_{n}-(p-q)\right\|\right)-g_{2}\left(\left\|u_{n}-v_{n}+(p-q)\right\|\right) \\
+2 \mu_{2}\left\|B_{2} p-B_{2} x_{n}\right\|\left\|u_{n}-q\right\|+2 \mu_{1}\left\|B_{1} q-B_{1} u_{n}\right\|\left\|v_{n}-p\right\| .
\end{gathered}
$$

By Lemma 2.2, we have from (3.16) and (3.21)

$$
\begin{aligned}
\| y_{n}- & p \|^{2} \\
\leq & \beta_{n}\left\|x_{n}-p\right\|^{2}+\gamma_{n}\left\|x_{n}-p\right\|^{2}+\delta_{n}\left\|v_{n}-p\right\|^{2} \\
\leq & \beta_{n}\left\|x_{n}-p\right\|^{2}+\gamma_{n}\left\|x_{n}-p\right\|^{2}+\delta_{n}\left[\left\|x_{n}-p\right\|^{2}-g_{1}\left(\left\|x_{n}-u_{n}-(p-q)\right\|\right)\right. \\
& -g_{2}\left(\left\|u_{n}-v_{n}+(p-q)\right\|\right)+2 \mu_{2}\left\|B_{2} p-B_{2} x_{n}\right\|\left\|u_{n}-q\right\| \\
& \left.+2 \mu_{1}\left\|B_{1} q-B_{1} u_{n}\right\|\left\|v_{n}-p\right\|\right] \\
\leq & \left\|x_{n}-p\right\|^{2}-\delta_{n}\left[g_{1}\left(\left\|x_{n}-u_{n}-(p-q)\right\|\right)+g_{2}\left(\left\|u_{n}-v_{n}+(p-q)\right\|\right)\right] \\
& +2 \mu_{2}\left\|B_{2} p-B_{2} x_{n}\right\|\left\|u_{n}-q\right\|+2 \mu_{1}\left\|B_{1} q-B_{1} u_{n}\right\|\left\|v_{n}-p\right\|,
\end{aligned}
$$

which hence leads to

$$
\begin{aligned}
& \delta_{n}\left[g_{1}\left(\left\|x_{n}-u_{n}-(p-q)\right\|\right)+g_{2}\left(\left\|u_{n}-v_{n}+(p-q)\right\|\right)\right] \\
& \quad \leq\left\|x_{n}-p\right\|^{2}-\left\|y_{n}-p\right\|^{2}+2 \mu_{2}\left\|B_{2} p-B_{2} x_{n}\right\|\left\|u_{n}-q\right\|+2 \mu_{1}\left\|B_{1} q-B_{1} u_{n}\right\|\left\|v_{n}-p\right\| \\
& \quad \leq\left(\left\|x_{n}-p\right\|+\left\|y_{n}-p\right\|\right)\left\|x_{n}-y_{n}\right\|+2 \mu_{2}\left\|B_{2} p-B_{2} x_{n}\right\|\left\|u_{n}-q\right\| \\
& \quad+2 \mu_{1}\left\|B_{1} q-B_{1} u_{n}\right\|\left\|v_{n}-p\right\| .
\end{aligned}
$$


From (3.18), (3.22), condition (ii) and the boundedness of $\left\{x_{n}\right\},\left\{y_{n}\right\},\left\{u_{n}\right\}$ and $\left\{v_{n}\right\}$, we deduce that

$$
\lim _{n \rightarrow \infty} g_{1}\left(\left\|x_{n}-u_{n}-(p-q)\right\|\right)=0 \quad \text { and } \quad \lim _{n \rightarrow \infty} g_{2}\left(\left\|u_{n}-v_{n}+(p-q)\right\|\right)=0
$$

Utilizing the properties of $g_{1}$ and $g_{2}$, we deduce that

$$
\lim _{n \rightarrow \infty}\left\|x_{n}-u_{n}-(p-q)\right\|=0 \text { and } \quad \lim _{n \rightarrow \infty}\left\|u_{n}-v_{n}+(p-q)\right\|=0 .
$$

From (3.23), we get

$$
\left\|x_{n}-v_{n}\right\| \leq\left\|x_{n}-u_{n}-(p-q)\right\|+\left\|u_{n}-v_{n}+(p-q)\right\| \rightarrow 0 \quad \text { as } n \rightarrow \infty .
$$

That is,

$$
\lim _{n \rightarrow \infty}\left\|x_{n}-G x_{n}\right\|=0
$$

Next, let us show that

$$
\lim _{n \rightarrow \infty}\left\|S_{n} x_{n}-x_{n}\right\|=0 \quad \text { and } \quad \lim _{n \rightarrow \infty}\left\|J_{r_{n}} x_{n}-x_{n}\right\|=0 .
$$

Indeed, utilizing Lemma 2.5 and (3.1), we have

$$
\begin{aligned}
\left\|y_{n}-p\right\|^{2}= & \left\|\delta_{n}\left(J_{r_{n}} G x_{n}-p\right)+\left(\beta_{n}+\gamma_{n}\right)\left(\frac{\beta_{n} x_{n}+\gamma_{n} S_{n} x_{n}}{\beta_{n}+\gamma_{n}}-p\right)\right\|^{2} \\
\leq & \delta_{n}\left\|J_{r_{n}} G x_{n}-p\right\|^{2}+\left(\beta_{n}+\gamma_{n}\right)\left\|\frac{\beta_{n} x_{n}+\gamma_{n} S_{n} x_{n}}{\beta_{n}+\gamma_{n}}-p\right\|^{2} \\
= & \delta_{n}\left\|J_{r_{n}} G x_{n}-p\right\|^{2}+\left(\beta_{n}+\gamma_{n}\right)\left\|\frac{\beta_{n}}{\beta_{n}+\gamma_{n}}\left(x_{n}-p\right)+\frac{\gamma_{n}}{\beta_{n}+\gamma_{n}}\left(S_{n} x_{n}-p\right)\right\|^{2} \\
\leq & \delta_{n}\left\|G x_{n}-p\right\|^{2}+\left(\beta_{n}+\gamma_{n}\right)\left[\frac{\beta_{n}}{\beta_{n}+\gamma_{n}}\left\|x_{n}-p\right\|^{2}+\frac{\gamma_{n}}{\beta_{n}+\gamma_{n}}\left\|S_{n} x_{n}-p\right\|^{2}\right. \\
& \left.-\frac{\beta_{n} \gamma_{n}}{\left(\beta_{n}+\gamma_{n}\right)^{2}} g_{3}\left(\left\|x_{n}-S_{n} x_{n}\right\|\right)\right] \\
\leq & \delta_{n}\left\|x_{n}-p\right\|^{2}+\beta_{n}\left\|x_{n}-p\right\|^{2}+\gamma_{n}\left\|x_{n}-p\right\|^{2}-\frac{\beta_{n} \gamma_{n}}{\beta_{n}+\gamma_{n}} g_{3}\left(\left\|x_{n}-S_{n} x_{n}\right\|\right) \\
= & \left\|x_{n}-p\right\|^{2}-\frac{\beta_{n} \gamma_{n}}{\beta_{n}+\gamma_{n}} g_{3}\left(\left\|x_{n}-S_{n} x_{n}\right\|\right),
\end{aligned}
$$

which immediately implies that

$$
\begin{aligned}
\beta_{n} \gamma_{n} g_{3}\left(\left\|x_{n}-S_{n} x_{n}\right\|\right) & \leq \frac{\beta_{n} \gamma_{n}}{\beta_{n}+\gamma_{n}} g_{3}\left(\left\|x_{n}-S_{n} x_{n}\right\|\right) \\
& \leq\left\|x_{n}-p\right\|^{2}-\left\|y_{n}-p\right\|^{2} \\
& \leq\left(\left\|x_{n}-p\right\|+\left\|y_{n}-p\right\|\right)\left\|x_{n}-y_{n}\right\| .
\end{aligned}
$$


So, from (3.12), the boundedness of $\left\{x_{n}\right\},\left\{y_{n}\right\}$ and conditions (ii), (v), it follows that

$$
\lim _{n \rightarrow \infty} g_{3}\left(\left\|x_{n}-S_{n} x_{n}\right\|\right)=0 .
$$

From the properties of $g_{3}$, we have

$$
\lim _{n \rightarrow \infty}\left\|x_{n}-S_{n} x_{n}\right\|=0 .
$$

Taking into account that

$$
y_{n}-x_{n}=\gamma_{n}\left(S_{n} x_{n}-x_{n}\right)+\delta_{n}\left(J_{r_{n}} G x_{n}-x_{n}\right),
$$

we have

$$
\begin{aligned}
\delta_{n}\left\|J_{r_{n}} G x_{n}-x_{n}\right\| & =\left\|y_{n}-x_{n}-\gamma_{n}\left(S_{n} x_{n}-x_{n}\right)\right\| \\
& \leq\left\|y_{n}-x_{n}\right\|+\gamma_{n}\left\|S_{n} x_{n}-x_{n}\right\| \\
& \leq\left\|y_{n}-x_{n}\right\|+\left\|S_{n} x_{n}-x_{n}\right\| .
\end{aligned}
$$

From (3.12), (3.25) and condition (ii), it follows that

$$
\lim _{n \rightarrow \infty}\left\|J_{r_{n}} G x_{n}-x_{n}\right\|=0 .
$$

Note that

$$
\left\|x_{n}-S x_{n}\right\| \leq\left\|x_{n}-S_{n} x_{n}\right\|+\left\|S_{n} x_{n}-S x_{n}\right\|
$$

So, in terms of (3.25) and Lemma 2.6, we have

$$
\lim _{n \rightarrow \infty}\left\|x_{n}-S x_{n}\right\|=0 .
$$

Also, note that

$$
\begin{aligned}
\left\|x_{n}-J_{r_{n}} x_{n}\right\| & \leq\left\|x_{n}-J_{r_{n}} G x_{n}\right\|+\left\|J_{r_{n}} G x_{n}-J_{r_{n}} x_{n}\right\| \\
& \leq\left\|x_{n}-J_{r_{n}} G x_{n}\right\|+\left\|G x_{n}-x_{n}\right\| .
\end{aligned}
$$

From (3.24) and (3.26), we have

$$
\lim _{n \rightarrow \infty}\left\|x_{n}-J_{r_{n}} x_{n}\right\|=0
$$

Furthermore, we claim that $\lim _{n \rightarrow \infty}\left\|x_{n}-J_{r} x_{n}\right\|=0$ for a fixed number $r$ such that $\varepsilon>$ $r>0$. In fact, taking into account the resolvent identity in Proposition 1.1, we have

$$
\begin{aligned}
\left\|J_{r_{n}} x_{n}-J_{r} x_{n}\right\| & =\left\|J_{r}\left(\frac{r}{r_{n}} x_{n}+\left(1-\frac{r}{r_{n}}\right) J_{r_{n}} x_{n}\right)-J_{r} x_{n}\right\| \\
& \leq\left(1-\frac{r}{r_{n}}\right)\left\|x_{n}-J_{r_{n}} x_{n}\right\| \\
& \leq\left\|x_{n}-J_{r_{n}} x_{n}\right\| .
\end{aligned}
$$


Thus, we get from (3.28) and (3.29)

$$
\begin{aligned}
\left\|x_{n}-J_{r} x_{n}\right\| & \leq\left\|x_{n}-J_{r_{n}} x_{n}\right\|+\left\|J_{r_{n}} x_{n}-J_{r} x_{n}\right\| \\
& \leq\left\|x_{n}-J_{r_{n}} x_{n}\right\|+\left\|x_{n}-J_{r_{n}} x_{n}\right\| \\
& =2\left\|x_{n}-J_{r_{n}} x_{n}\right\| \rightarrow 0 \quad \text { as } n \rightarrow \infty .
\end{aligned}
$$

That is,

$$
\lim _{n \rightarrow \infty}\left\|x_{n}-J_{r} x_{n}\right\|=0
$$

Define a mapping $W x=\left(1-\theta_{1}-\theta_{2}\right) J_{r} x+\theta_{1} S x+\theta_{2} G x$, where $\theta_{1}, \theta_{2} \in(0,1)$ are two constants with $\theta_{1}+\theta_{2}<1$. Then by Lemma 2.8, we have that $\operatorname{Fix}(W)=\operatorname{Fix}\left(J_{r}\right) \cap \operatorname{Fix}(S) \cap \operatorname{Fix}(G)=F$. We observe that

$$
\begin{aligned}
\left\|x_{n}-W x_{n}\right\| & =\left\|\left(1-\theta_{1}-\theta_{2}\right)\left(x_{n}-J_{r} x_{n}\right)+\theta_{1}\left(x_{n}-S x_{n}\right)+\theta_{2}\left(x_{n}-G x_{n}\right)\right\| \\
& \leq\left(1-\theta_{1}-\theta_{2}\right)\left\|x_{n}-J_{r} x_{n}\right\|+\theta_{1}\left\|x_{n}-S x_{n}\right\|+\theta_{2}\left\|x_{n}-G x_{n}\right\| .
\end{aligned}
$$

From (3.24), (3.27) and (3.30), we obtain

$$
\lim _{n \rightarrow \infty}\left\|x_{n}-W x_{n}\right\|=0
$$

Now, we claim that

$$
\limsup _{n \rightarrow \infty}\left\langle f(q)-q, J\left(x_{n}-q\right)\right\rangle \leq 0
$$

where $q=s-\lim _{t \rightarrow 0} x_{t}$ with $x_{t}$ being the fixed point of the contraction

$$
x \mapsto t f(x)+(1-t) W x .
$$

Then $x_{t}$ solves the fixed point equation $x_{t}=t f\left(x_{t}\right)+(1-t) W x_{t}$. Thus, we have

$$
x_{t}-x_{n}=(1-t)\left(W x_{t}-x_{n}\right)+t\left(f\left(x_{t}\right)-x_{n}\right) \text {. }
$$

By Lemma 2.2, we conclude that

$$
\begin{aligned}
\| x_{t}- & x_{n} \|^{2} \\
= & \left\|(1-t)\left(W x_{t}-x_{n}\right)+t\left(f\left(x_{t}\right)-x_{n}\right)\right\|^{2} \\
\leq & (1-t)^{2}\left\|W x_{t}-x_{n}\right\|^{2}+2 t\left\langle f\left(x_{t}\right)-x_{n}, J\left(x_{t}-x_{n}\right)\right\rangle \\
\leq & (1-t)^{2}\left(\left\|W x_{t}-W x_{n}\right\|+\left\|W x_{n}-x_{n}\right\|\right)^{2}+2 t\left\langle f\left(x_{t}\right)-x_{n}, J\left(x_{t}-x_{n}\right)\right\rangle \\
\leq & (1-t)^{2}\left(\left\|x_{t}-x_{n}\right\|+\left\|W x_{n}-x_{n}\right\|\right)^{2}+2 t\left\langle f\left(x_{t}\right)-x_{n}, J\left(x_{t}-x_{n}\right)\right\rangle \\
= & (1-t)^{2}\left[\left\|x_{t}-x_{n}\right\|^{2}+2\left\|x_{t}-x_{n}\right\|\left\|W x_{n}-x_{n}\right\|+\left\|W x_{n}-x_{n}\right\|^{2}\right] \\
& +2 t\left\langle f\left(x_{t}\right)-x_{t}, J\left(x_{t}-x_{n}\right)\right\rangle+2 t\left\langle x_{t}-x_{n}, J\left(x_{t}-x_{n}\right)\right\rangle
\end{aligned}
$$




$$
\begin{aligned}
= & \left(1-2 t+t^{2}\right)\left\|x_{t}-x_{n}\right\|^{2}+f_{n}(t) \\
& +2 t\left\langle f\left(x_{t}\right)-x_{t}, J\left(x_{t}-x_{n}\right)\right\rangle+2 t\left\|x_{t}-x_{n}\right\|^{2},
\end{aligned}
$$

where

$$
f_{n}(t)=(1-t)^{2}\left(2\left\|x_{t}-x_{n}\right\|+\left\|x_{n}-W x_{n}\right\|\right)\left\|x_{n}-W x_{n}\right\| \rightarrow 0, \quad \text { as } n \rightarrow \infty .
$$

It follows from (3.33) that

$$
\left\langle x_{t}-f\left(x_{t}\right), J\left(x_{t}-x_{n}\right)\right\rangle \leq \frac{t}{2}\left\|x_{t}-x_{n}\right\|^{2}+\frac{1}{2 t} f_{n}(t) .
$$

Letting $n \rightarrow \infty$ in (3.35) and noticing (3.34), we derive

$$
\limsup _{n \rightarrow \infty}\left\langle x_{t}-f\left(x_{t}\right), J\left(x_{t}-x_{n}\right)\right\rangle \leq \frac{t}{2} M_{2}
$$

where $M_{2}>0$ is a constant such that $\left\|x_{t}-x_{n}\right\|^{2} \leq M_{2}$ for all $t \in(0,1)$ and $n \geq 0$. Taking $t \rightarrow 0$ in (3.36), we have

$$
\limsup _{t \rightarrow 0} \limsup _{n \rightarrow \infty}\left\langle x_{t}-f\left(x_{t}\right), J\left(x_{t}-x_{n}\right)\right\rangle \leq 0 .
$$

On the other hand, we have

$$
\begin{aligned}
\langle f(q) & \left.-q, J\left(x_{n}-q\right)\right\rangle \\
= & \left\langle f(q)-q, J\left(x_{n}-q\right)\right\rangle-\left\langle f(q)-q, J\left(x_{n}-x_{t}\right)\right\rangle+\left\langle f(q)-q, J\left(x_{n}-x_{t}\right)\right\rangle \\
& -\left\langle f(q)-x_{t}, J\left(x_{n}-x_{t}\right)\right\rangle+\left\langle f(q)-x_{t}, J\left(x_{n}-x_{t}\right)\right\rangle-\left\langle f\left(x_{t}\right)-x_{t}, J\left(x_{n}-x_{t}\right)\right\rangle \\
& +\left\langle f\left(x_{t}\right)-x_{t}, J\left(x_{n}-x_{t}\right)\right\rangle \\
= & \left\langle f(q)-q, J\left(x_{n}-q\right)-J\left(x_{n}-x_{t}\right)\right\rangle+\left\langle x_{t}-q, J\left(x_{n}-x_{t}\right)\right\rangle \\
& +\left\langle f(q)-f\left(x_{t}\right), J\left(x_{n}-x_{t}\right)\right\rangle+\left\langle f\left(x_{t}\right)-x_{t}, J\left(x_{n}-x_{t}\right)\right\rangle .
\end{aligned}
$$

It follows that

$$
\begin{aligned}
\limsup _{n \rightarrow \infty}\left\langle f(q)-q, J\left(x_{n}-q\right)\right\rangle \leq & \limsup _{n \rightarrow \infty}\left\langle f(q)-q, J\left(x_{n}-q\right)-J\left(x_{n}-x_{t}\right)\right\rangle \\
& +\left\|x_{t}-q\right\| \limsup _{n \rightarrow \infty}\left\|x_{n}-x_{t}\right\|+\rho\left\|q-x_{t}\right\| \limsup _{n \rightarrow \infty}\left\|x_{n}-x_{t}\right\| \\
& +\limsup _{n \rightarrow \infty}\left\langle f\left(x_{t}\right)-x_{t}, J\left(x_{n}-x_{t}\right)\right\rangle .
\end{aligned}
$$

Taking into account that $x_{t} \rightarrow q$ as $t \rightarrow 0$, we have

$$
\begin{aligned}
\limsup _{n \rightarrow \infty}\left\langle f(q)-q, J\left(x_{n}-q\right)\right\rangle & =\limsup _{t \rightarrow 0} \limsup _{n \rightarrow \infty}\left\langle f(q)-q, J\left(x_{n}-q\right)\right\rangle \\
& \leq \limsup _{t \rightarrow 0} \limsup _{n \rightarrow \infty}\left\langle f(q)-q, J\left(x_{n}-q\right)-J\left(x_{n}-x_{t}\right)\right\rangle .
\end{aligned}
$$


Since $X$ has a uniformly Frechet differentiable norm, the duality mapping $J$ is norm-tonorm uniformly continuous on bounded subsets of $X$. Consequently, the two limits are interchangeable, and hence (3.32) holds. From (3.4), we get $\left(x_{n+1}-q\right)-\left(x_{n}-q\right) \rightarrow 0$. Noticing the norm-to-norm uniform continuity of $J$ on bounded subsets of $X$, we deduce from (3.32) that

$$
\begin{aligned}
& \limsup _{n \rightarrow \infty}\left\langle f(q)-q, J\left(x_{n+1}-q\right)\right\rangle \\
& \quad=\limsup _{n \rightarrow \infty}\left(\left\langle f(q)-q, J\left(x_{n}-q\right)\right\rangle+\left\langle f(q)-q, J\left(x_{n+1}-q\right)-J\left(x_{n}-q\right)\right\rangle\right) \\
& \quad=\limsup _{n \rightarrow \infty}\left\langle f(q)-q, J\left(x_{n}-q\right)\right\rangle \leq 0 .
\end{aligned}
$$

Finally, let us show that $x_{n} \rightarrow q$ as $n \rightarrow \infty$. Utilizing Lemma 2.2, from (3.1) and the convexity of $\|\cdot\|^{2}$, we get

$$
\begin{aligned}
\left\|y_{n}-q\right\|^{2} & \leq \beta_{n}\left\|x_{n}-q\right\|^{2}+\gamma_{n}\left\|S_{n} x_{n}-q\right\|^{2}+\delta_{n}\left\|J_{r_{n}} G x_{n}-q\right\|^{2} \\
& \leq \beta_{n}\left\|x_{n}-q\right\|^{2}+\gamma_{n}\left\|x_{n}-q\right\|^{2}+\delta_{n}\left\|x_{n}-q\right\|^{2} \\
& =\left\|x_{n}-q\right\|^{2},
\end{aligned}
$$

and

$$
\begin{aligned}
\left\|x_{n+1}-q\right\|^{2} & =\left\|\alpha_{n}\left(f\left(x_{n}\right)-f(q)\right)+\left(1-\alpha_{n}\right)\left(y_{n}-q\right)+\alpha_{n}(f(q)-q)\right\|^{2} \\
& \leq\left\|\alpha_{n}\left(f\left(x_{n}\right)-f(q)\right)+\left(1-\alpha_{n}\right)\left(y_{n}-q\right)\right\|^{2}+2 \alpha_{n}\left\langle f(q)-q, J\left(x_{n+1}-q\right)\right\rangle \\
& \leq \alpha_{n}\left\|f\left(x_{n}\right)-f(q)\right\|^{2}+\left(1-\alpha_{n}\right)\left\|y_{n}-q\right\|^{2}+2 \alpha_{n}\left\langle f(q)-q, J\left(x_{n+1}-q\right)\right\rangle \\
& \leq \alpha_{n} \rho\left\|x_{n}-q\right\|^{2}+\left(1-\alpha_{n}\right)\left\|x_{n}-q\right\|^{2}+2 \alpha_{n}\left\langle f(q)-q, J\left(x_{n+1}-q\right)\right\rangle \\
& =\left(1-\alpha_{n}(1-\rho)\right)\left\|x_{n}-q\right\|^{2}+2 \alpha_{n}\left\langle f(q)-q, J\left(x_{n+1}-q\right)\right\rangle \\
& =\left(1-\alpha_{n}(1-\rho)\right)\left\|x_{n}-q\right\|^{2}+\alpha_{n}(1-\rho) \frac{2\left\langle f(q)-q, J\left(x_{n+1}-q\right)\right\rangle}{1-\rho} .
\end{aligned}
$$

Applying Lemma 2.1 to (3.39), we obtain that $x_{n} \rightarrow q$ as $n \rightarrow \infty$. This completes the proof.

Corollary 3.1 Let $C$ be a nonempty closed convex subset of a uniformly convex and 2uniformly smooth Banach space $X$. Let $\Pi_{C}$ be a sunny nonexpansive retraction from $X$ onto C. Let $A \subset X \times X$ be an accretive operator in $X$ such that $\overline{D(A)} \subset C \subset \bigcap_{r>0} R(I+r A)$. Let $V: C \rightarrow C$ be an $\alpha$-strictly pseudocontractive mapping. Let $f: C \rightarrow C$ be a contraction with coefficient $\rho \in(0,1)$. Let $\left\{S_{i}\right\}_{i=0}^{\infty}$ be a countable family of nonexpansive mappings of $C$ into itself such that $F=\bigcap_{i=0}^{\infty} \operatorname{Fix}\left(S_{i}\right) \cap \operatorname{Fix}(V) \cap A^{-1} 0 \neq \emptyset$. For arbitrarily given $x_{0} \in C$, let $\left\{x_{n}\right\}$ be the sequence generated by

$$
\left\{\begin{array}{l}
y_{n}=\beta_{n} x_{n}+\gamma_{n} S_{n} x_{n}+\delta_{n} J_{r_{n}}((1-l) I+l V) x_{n}, \\
x_{n+1}=\alpha_{n} f\left(x_{n}\right)+\left(1-\alpha_{n}\right) y_{n}, \quad \forall n \geq 0,
\end{array}\right.
$$

where $0<l<\frac{\alpha}{\kappa^{2}}$, and $\left\{\alpha_{n}\right\},\left\{\beta_{n}\right\},\left\{\gamma_{n}\right\}$ and $\left\{\delta_{n}\right\}$ are the sequences in $[0,1]$ such that $\beta_{n}+\gamma_{n}+$ $\delta_{n}=1$ for all $n \geq 0$. Suppose that the following conditions hold: 
(i) $\sum_{n=0}^{\infty} \alpha_{n}=\infty$ and $0 \leq \alpha_{n} \leq 1-\rho, \forall n \geq n_{0}$ for some integer $n_{0} \geq 0$;

(ii) $\liminf _{n \rightarrow \infty} \gamma_{n}>0$ and $\liminf _{n \rightarrow \infty} \delta_{n}>0$;

(iii) $\lim _{n \rightarrow \infty}\left(\left|\frac{\alpha_{n+1}}{1-\left(1-\alpha_{n+1}\right) \beta_{n+1}}-\frac{\alpha_{n}}{1-\left(1-\alpha_{n}\right) \beta_{n}}\right|+\left|\frac{\delta_{n+1}}{1-\beta_{n+1}}-\frac{\delta_{n}}{1-\beta_{n}}\right|\right)=0$;

(iv) $\lim _{n \rightarrow \infty}\left|r_{n+1}-r_{n}\right|=0$ and $r_{n} \geq \varepsilon>0$ for all $n \geq 0$;

(v) $0<\liminf _{n \rightarrow \infty} \beta_{n} \leq \limsup _{n \rightarrow \infty} \beta_{n}<1$.

Assume that $\sum_{n=0}^{\infty} \sup _{x \in D}\left\|S_{n+1} x-S_{n} x\right\|<\infty$ for any bounded subset $D$ of $C$, and let $S$ be a mapping of $C$ into itself defined by $S x=\lim _{n \rightarrow \infty} S_{n} x$ for all $x \in C$, and suppose that $\operatorname{Fix}(S)=$ $\bigcap_{i=0}^{\infty} \operatorname{Fix}\left(S_{i}\right)$. Then

$$
x_{n} \rightarrow q \Longleftrightarrow \alpha_{n}\left(f\left(x_{n}\right)-x_{n}\right) \rightarrow 0,
$$

where $q \in F$ solves the following VIP:

$$
\langle q-f(q), J(q-p)\rangle \leq 0, \quad \forall p \in F .
$$

Proof In Theorem 3.1, we put $B_{1}=I-V, B_{2}=0$ and $\mu_{1}=l$, where $0<l<\frac{\alpha}{\kappa^{2}}$. Then GSVI (1.1) is equivalent to the VIP of finding $x^{*} \in C$ such that

$$
\left\langle B_{1} x^{*}, J\left(x-x^{*}\right)\right| \geq 0, \quad \forall x \in C .
$$

In this case, $B_{1}: C \rightarrow X$ is $\alpha$-inverse strongly accretive. It is not hard to see that $\operatorname{Fix}(V)=$ $\mathrm{VI}\left(C, B_{1}\right)$. As a matter of fact, we have, for $l>0$,

$$
\begin{aligned}
u \in \operatorname{VI}\left(C, B_{1}\right) & \Leftrightarrow\left\langle B_{1} u, J(y-u)\right| \geq 0, \quad \forall y \in C \\
& \Leftrightarrow \quad\left\langle u-l B_{1} u-u, J(u-y)\right| \geq 0, \quad \forall y \in C \\
& \Leftrightarrow \quad u=\Pi_{C}\left(u-l B_{1} u\right) \\
& \Leftrightarrow \quad u=\Pi_{C}(u-l u+l V u) \\
& \Leftrightarrow \quad\langle u-l u+l V u-u, J(u-y)| \geq 0, \quad \forall y \in C \\
& \Leftrightarrow \quad\langle u-V u, J(u-y)\rangle \leq 0, \quad \forall y \in C \\
& \Leftrightarrow u=V u \\
& \Leftrightarrow u \in \operatorname{Fix}(V) .
\end{aligned}
$$

Accordingly, we know that $F=\bigcap_{i=0}^{\infty} \operatorname{Fix}\left(S_{i}\right) \cap \Omega \cap A^{-1} 0=\bigcap_{i=0}^{\infty} \operatorname{Fix}\left(S_{i}\right) \cap \operatorname{Fix}(V) \cap A^{-1} 0$, and

$$
\begin{aligned}
& \Pi_{C}\left(I-\mu_{1} B_{1}\right) \Pi_{C}\left(I-\mu_{2} B_{2}\right) x_{n} \\
& \quad=\Pi_{C}\left(I-\mu_{1} B_{1}\right) x_{n} \\
& \quad=\Pi_{C}\left((1-l) x_{n}+l V x_{n}\right)=((1-l) I+l V) x_{n} .
\end{aligned}
$$

So, the scheme (3.1) reduces to (3.40). Therefore, the desired result follows from Theorem 3.1 .

Remark 3.1 Theorem 3.1 improves, extends, supplements and develops Jung [22, Theorem 3.1], Ceng and Yao [2, Theorem 3.1] and Cai and Bu [3, Theorem 3.1] in the following aspects. 
(i) The problem of finding a point $q \in \bigcap_{i=0}^{\infty} \operatorname{Fix}\left(S_{i}\right) \cap \Omega \cap A^{-1} 0$ in our Theorem 3.1 is more general and more subtle than any of the problems of finding a point $q \in A^{-1} 0$ in [22, Theorem 3.1], the problem of finding a point $q \in \operatorname{Fix}(T)$ in [2, Theorem 3.1], and the problem of finding a point $q \in \bigcap_{i=1}^{\infty} \operatorname{Fix}\left(T_{i}\right) \cap \Omega$ in [3, Theorem 3.1].

(ii) The iterative scheme in [2, Theorem 3.1] is extended to develop the iterative scheme (3.1) of Theorem 3.1 by virtue of the iterative schemes of [22, Theorem 3.1] and [3, Theorem 3.1]. The iterative scheme (3.1) of Theorem 3.1 is more advantageous and more flexible than the iterative scheme of [2, Theorem 3.1], because it can be applied to solving three problems (i.e., GSVI (1.1), fixed point problem and zero point problem) and involves several parameter sequences $\left\{r_{n}\right\},\left\{\alpha_{n}\right\},\left\{\beta_{n}\right\},\left\{\gamma_{n}\right\}$ and $\left\{\delta_{n}\right\}$.

(iii) Our Theorem 3.1 extends and generalizes Ceng and Yao [2, Theorem 3.1] from a nonexpansive mapping to a countable family of nonexpansive mappings, and Jung [22, Theorems 3.1] to the setting of a countable family of nonexpansive mappings and GSVI (1.1) for two inverse-strongly accretive mappings. In the meantime, our Theorem $3.1 \mathrm{ex}-$ tends and generalizes $\mathrm{Cai}$ and $\mathrm{Bu}$ [3, Theorem 3.1] to the setting of an accretive operator.

(iv) The iterative scheme (3.1) in Theorem 3.1 is very different from any in [22, Theorem 3.1], [2, Theorem 3.1] and [3, Theorem 3.1], because the mapping $G$ in [3, Theorem 3.1] and the mapping $J_{r_{n}}$ in [22, Theorem 3.1] are replaced by the same composite mapping $J_{r_{n}} G$ in the iterative scheme (3.1) of our Theorem 3.1.

(v) Cai and Bu's proof in [3, Theorem 3.1] depends on the argument techniques in [6], the inequality in 2-uniformly smooth Banach spaces (see Proposition 2.1) and the inequality in smooth and uniform convex Banach spaces (see Proposition 2.2). Because the composite mapping $J_{r_{n}} G$ appears in the iterative scheme (3.1) of our Theorem 3.1, the proof of our Theorem 3.1 depends on the argument techniques in [6], the inequality in 2-uniformly smooth Banach spaces (see Proposition 2.1), the inequality in smooth and uniform convex Banach spaces (see Proposition 2.2), the inequalities in uniform convex Banach spaces (see Lemmas 2.5 and 2.9 in Section 2 of this paper), and the resolvent identity for accretive operators (see Proposition 1.1).

(vi) It is worth emphasizing that the assumption of asymptotic regularity on $\left\{x_{n}\right\}$ in [22, Theorem 3.1] is dropped by Theorem 3.1, and there is no assumption of the convergence of parameter sequences to zero in our Theorem 3.1.

\section{Composite Mann iterative algorithms in uniformly convex Banach spaces having uniformly Gâteaux differentiable norms}

In this section, we introduce our composite Mann iterative algorithms in uniformly convex Banach spaces having uniformly Gâteaux differentiable norms and show the strong convergence theorems. First, we give some useful lemmas whose proofs will be omitted because they can be obtained by standard argument.

Lemma 4.1 Let $C$ be a nonempty closed convex subset of a smooth Banach space $X$, and let the mapping $B_{i}: C \rightarrow X$ be $\lambda_{i}$-strictly pseudocontractive and $\alpha_{i}$-strongly accretive with $\alpha_{i}+\lambda_{i} \geq 1$ for $i=1,2$. Then, for $\mu_{i} \in(0,1]$ we have

$$
\left\|\left(I-\mu_{i} B_{i}\right) x-\left(I-\mu_{i} B_{i}\right) y\right\| \leq\left\{\sqrt{\frac{1-\alpha_{i}}{\lambda_{i}}}+\left(1-\mu_{i}\right)\left(1+\frac{1}{\lambda_{i}}\right)\right\}\|x-y\|, \quad \forall x, y \in C,
$$


for $i=1$, 2. In particular, if $1-\frac{\lambda_{i}}{1+\lambda_{i}}\left(1-\sqrt{\frac{1-\alpha_{i}}{\lambda_{i}}}\right) \leq \mu_{i} \leq 1$, then $I-\mu_{i} B_{i}$ is nonexpansive for $i=1,2$.

Lemma 4.2 Let $C$ be a nonempty closed convex subset of a smooth Banach space $X$. Let $\Pi_{C}$ be a sunny nonexpansive retraction from $X$ onto $C$, and let the mapping $B_{i}: C \rightarrow X$ be $\lambda_{i}$-strictly pseudocontractive and $\alpha_{i}$-strongly accretive with $\alpha_{i}+\lambda_{i} \geq 1$ for $i=1$, 2 . Let $G: C \rightarrow C$ be the mapping defined by

$$
G(x)=\Pi_{C}\left[\Pi_{C}\left(x-\mu_{2} B_{2} x\right)-\mu_{1} B_{1} \Pi_{C}\left(x-\mu_{2} B_{2} x\right)\right], \quad \forall x \in C .
$$

If $1-\frac{\lambda_{i}}{1+\lambda_{i}}\left(1-\sqrt{\frac{1-\alpha_{i}}{\lambda_{i}}}\right) \leq \mu_{i} \leq 1$, then $G: C \rightarrow C$ is nonexpansive.

We now state and prove the main result of this section.

Theorem 4.1 Let $C$ be a nonempty closed convex subset of a uniformly convex Banach space $X$ which has a uniformly Gâteaux differentiable norm. Let $\Pi_{C}$ be a sunny nonexpansive retraction from $X$ onto $C$. Let $A \subset X \times X$ be an accretive operator in $X$ such that $\overline{D(A)} \subset C \subset \bigcap_{r>0} R(I+r A)$. Let $B_{i}: C \rightarrow X$ be $\lambda_{i}$-strictly pseudocontractive and $\alpha_{i}$ strongly accretive with $\lambda_{i}+\alpha_{i} \geq 1$ for $i=1$, 2. Let $f: C \rightarrow C$ be a contraction with coefficient $\rho \in(0,1)$. Let $\left\{S_{i}\right\}_{i=0}^{\infty}$ be a countable family of nonexpansive mappings of $C$ into itself such that $F=\bigcap_{i=0}^{\infty} \operatorname{Fix}\left(S_{i}\right) \cap \Omega \cap A^{-1} 0 \neq \emptyset$, where $\Omega$ is the fixed point set of the mapping $G=\Pi_{C}\left(I-\mu_{1} B_{1}\right) \Pi_{C}\left(I-\mu_{2} B_{2}\right)$ with $1-\frac{\lambda_{i}}{1+\lambda_{i}}\left(1-\sqrt{\frac{1-\alpha_{i}}{\lambda_{i}}}\right) \leq \mu_{i} \leq 1$ for $i=1$, 2. For arbitrarily given $x_{0} \in C$, let $\left\{x_{n}\right\}$ be the sequence generated by

$$
\left\{\begin{array}{l}
y_{n}=\alpha_{n} f\left(x_{n}\right)+\beta_{n} x_{n}+\gamma_{n} S_{n} x_{n}+\delta_{n} J_{r_{n}} G x_{n}, \\
x_{n+1}=\sigma_{n} G x_{n}+\left(1-\sigma_{n}\right) y_{n}, \quad \forall n \geq 0,
\end{array}\right.
$$

where $\left\{\alpha_{n}\right\},\left\{\beta_{n}\right\},\left\{\gamma_{n}\right\},\left\{\delta_{n}\right\}$ and $\left\{\sigma_{n}\right\}$ are the sequences in $(0,1)$ such that $\alpha_{n}+\beta_{n}+\gamma_{n}+\delta_{n}=1$ for all $n \geq 0$. Suppose that the following conditions hold:

(i) $\lim _{n \rightarrow \infty} \alpha_{n}=0$ and $\sum_{n=0}^{\infty} \alpha_{n}=\infty$;

(ii) $\left\{\gamma_{n}\right\},\left\{\delta_{n}\right\} \subset[c, d]$ for some $c, d \in(0,1)$;

(iii) $\sum_{n=1}^{\infty}\left(\left|\sigma_{n}-\sigma_{n-1}\right|+\left|\alpha_{n}-\alpha_{n-1}\right|+\left|\beta_{n}-\beta_{n-1}\right|+\left|\gamma_{n}-\gamma_{n-1}\right|+\left|\delta_{n}-\delta_{n-1}\right|\right)<\infty$;

(iv) $\sum_{n=1}^{\infty}\left|r_{n}-r_{n-1}\right|<\infty$ and $r_{n} \geq \varepsilon>0$ for all $n \geq 0$;

(v) $0<\liminf _{n \rightarrow \infty} \beta_{n} \leq \limsup \sup _{n \rightarrow \infty} \beta_{n}<1$ and $0<\liminf _{n \rightarrow \infty} \sigma_{n} \leq \lim \sup _{n \rightarrow \infty} \sigma_{n}<1$. Assume that $\sum_{n=1}^{\infty} \sup _{x \in D}\left\|S_{n} x-S_{n-1} x\right\|<\infty$ for any bounded subset $D$ of $C$, and let $S$ be a mapping of $C$ into itself defined by $S x=\lim _{n \rightarrow \infty} S_{n} x$ for all $x \in C$, and suppose that $\operatorname{Fix}(S)=$ $\bigcap_{i=0}^{\infty} \operatorname{Fix}\left(S_{i}\right)$. Then $\left\{x_{n}\right\}$ converges strongly to $q \in F$, which solves the following VIP:

$$
\langle q-f(q), J(q-p)\rangle \leq 0, \quad \forall p \in F .
$$

Proof First of all, take a fixed $p \in F$ arbitrarily. Then we obtain $p=G p, p=S_{n} p$ and $J_{r_{n}} p=p$ for all $n \geq 0$. By Lemma 4.2, we get from (4.1)

$$
\begin{aligned}
\left\|y_{n}-p\right\| & \leq \alpha_{n}\left\|f\left(x_{n}\right)-p\right\|+\beta_{n}\left\|x_{n}-p\right\|+\gamma_{n}\left\|S_{n} x_{n}-p\right\|+\delta_{n}\left\|J_{r_{n}} G x_{n}-p\right\| \\
& \leq \alpha_{n}\left(\rho\left\|x_{n}-p\right\|+\|f(p)-p\|\right)+\beta_{n}\left\|x_{n}-p\right\|+\gamma_{n}\left\|x_{n}-p\right\|+\delta_{n}\left\|x_{n}-p\right\| \\
& =\left(1-\alpha_{n}(1-\rho)\right)\left\|x_{n}-p\right\|+\alpha_{n}\|f(p)-p\|,
\end{aligned}
$$


and hence

$$
\begin{aligned}
\left\|x_{n+1}-p\right\| & \leq \sigma_{n}\left\|G x_{n}-p\right\|+\left(1-\sigma_{n}\right)\left\|y_{n}-p\right\| \\
& \leq \sigma_{n}\left\|x_{n}-p\right\|+\left(1-\sigma_{n}\right)\left[\left(1-\alpha_{n}(1-\rho)\right)\left\|x_{n}-p\right\|+\alpha_{n}\|f(p)-p\|\right] \\
& =\left(1-\left(1-\sigma_{n}\right) \alpha_{n}(1-\rho)\right)\left\|x_{n}-p\right\|+\left(1-\sigma_{n}\right) \alpha_{n}\|f(p)-p\| \\
& =\left(1-\left(1-\sigma_{n}\right) \alpha_{n}(1-\rho)\right)\left\|x_{n}-p\right\|+\left(1-\sigma_{n}\right) \alpha_{n}(1-\rho) \frac{\|f(p)-p\|}{1-\rho} \\
& \leq \max \left\{\left\|x_{n}-p\right\|, \frac{\|f(p)-p\|}{1-\rho}\right\} .
\end{aligned}
$$

By induction, we have

$$
\left\|x_{n}-p\right\| \leq \max \left\{\left\|x_{0}-p\right\|, \frac{\|f(p)-p\|}{1-\rho}\right\}, \quad \forall n \geq 0,
$$

which implies that $\left\{x_{n}\right\}$ is bounded and so are the sequences $\left\{y_{n}\right\},\left\{G x_{n}\right\},\left\{f\left(x_{n}\right)\right\}$.

Let us show that

$$
\lim _{n \rightarrow \infty}\left\|x_{n+1}-x_{n}\right\|=0
$$

As a matter of fact, observe that $y_{n}$ can be rewritten as follows

$$
y_{n}=\beta_{n} x_{n}+\left(1-\beta_{n}\right) z_{n},
$$

where $z_{n}=\frac{\alpha_{n} f\left(x_{n}\right)+\gamma_{n} S_{n} x_{n}+\delta_{n} J_{n} G x_{n}}{1-\beta_{n}}$. Observe that

$$
\begin{aligned}
\| z_{n}- & z_{n-1} \| \\
= & \left\|\frac{\alpha_{n} f\left(x_{n}\right)+\gamma_{n} S_{n} x_{n}+\delta_{n} J_{r_{n}} G x_{n}}{1-\beta_{n}}-\frac{\alpha_{n-1} f\left(x_{n-1}\right)+\gamma_{n-1} S_{n-1} x_{n-1}+\delta_{n-1} J_{r_{n-1}} G x_{n-1}}{1-\beta_{n-1}}\right\| \\
= & \left\|\frac{y_{n}-\beta_{n} x_{n}}{1-\beta_{n}}-\frac{y_{n-1}-\beta_{n-1} x_{n-1}}{1-\beta_{n-1}}\right\| \\
= & \left\|\frac{y_{n}-\beta_{n} x_{n}}{1-\beta_{n}}-\frac{y_{n-1}-\beta_{n-1} x_{n-1}}{1-\beta_{n}}+\frac{y_{n-1}-\beta_{n-1} x_{n-1}}{1-\beta_{n}}-\frac{y_{n-1}-\beta_{n-1} x_{n-1}}{1-\beta_{n-1}}\right\| \\
\leq & \left\|\frac{y_{n}-\beta_{n} x_{n}}{1-\beta_{n}}-\frac{y_{n-1}-\beta_{n-1} x_{n-1}}{1-\beta_{n}}\right\|+\left\|\frac{y_{n-1}-\beta_{n-1} x_{n-1}}{1-\beta_{n}}-\frac{y_{n-1}-\beta_{n-1} x_{n-1}}{1-\beta_{n-1}}\right\| \\
= & \frac{1}{1-\beta_{n}}\left\|y_{n}-\beta_{n} x_{n}-\left(y_{n-1}-\beta_{n-1} x_{n-1}\right)\right\|+\left|\frac{1}{1-\beta_{n}}-\frac{1}{1-\beta_{n-1}}\right|\left\|y_{n-1}-\beta_{n-1} x_{n-1}\right\| \\
= & \frac{1}{1-\beta_{n}}\left\|y_{n}-\beta_{n} x_{n}-\left(y_{n-1}-\beta_{n-1} x_{n-1}\right)\right\|+\frac{\left|\beta_{n}-\beta_{n-1}\right|}{\left(1-\beta_{n-1}\right)\left(1-\beta_{n}\right)}\left\|y_{n-1}-\beta_{n-1} x_{n-1}\right\| \\
= & \frac{1}{1-\beta_{n}}\left\|\alpha_{n} f\left(x_{n}\right)+\gamma_{n} S_{n} x_{n}+\delta_{n} J_{r_{n}} G x_{n}-\alpha_{n-1} f\left(x_{n-1}\right)-\gamma_{n-1} S_{n-1} x_{n-1}-\delta_{n-1} J_{r_{n-1}} G x_{n-1}\right\| \\
& +\frac{\left|\beta_{n}-\beta_{n-1}\right|}{\left(1-\beta_{n-1}\right)\left(1-\beta_{n}\right)}\left\|y_{n-1}-\beta_{n-1} x_{n-1}\right\| \\
\leq & \frac{1}{1-\beta_{n}}\left[\alpha_{n}\left\|f\left(x_{n}\right)-f\left(x_{n-1}\right)\right\|+\gamma_{n}\left\|S_{n} x_{n}-S_{n-1} x_{n-1}\right\|+\delta_{n}\left\|J r_{n} G x_{n}-J_{r_{n-1}} G x_{n-1}\right\|\right.
\end{aligned}
$$




$$
\begin{aligned}
& \left.+\left|\alpha_{n}-\alpha_{n-1}\right|\left\|f\left(x_{n-1}\right)\right\|+\left|\gamma_{n}-\gamma_{n-1}\right|\left\|S_{n-1} x_{n-1}\right\|+\left|\delta_{n}-\delta_{n-1}\right||| J_{r_{n-1}} G x_{n-1} \|\right] \\
& +\frac{\left|\beta_{n}-\beta_{n-1}\right|}{\left(1-\beta_{n-1}\right)\left(1-\beta_{n}\right)}\left\|y_{n-1}-\beta_{n-1} x_{n-1}\right\| .
\end{aligned}
$$

On the other hand, repeating the same arguments as those of (3.8) in the proof of Theorem 3.1, we can deduce that for all $n \geq 0$,

$$
\left\|J_{r_{n+1}} G x_{n+1}-J_{r_{n}} G x_{n}\right\| \leq\left\|x_{n}-x_{n+1}\right\|+M_{0}\left|r_{n}-r_{n+1}\right|, \quad \forall n \geq 0,
$$

where $\sup _{n \geq 0}\left\{\frac{1}{\varepsilon}\left(\left\|J_{r_{n+1}} G x_{n+1}-G x_{n}\right\|+\left\|J_{r_{n}} G x_{n}-G x_{n+1}\right\|\right)\right\} \leq M_{0}$ for some $M_{0}>0$. Taking into account $0<\liminf _{n \rightarrow \infty} \beta_{n} \leq \lim \sup _{n \rightarrow \infty} \beta_{n}<1$, we may assume, without loss of generality, that $\left\{\beta_{n}\right\} \subset[\hat{c}, \hat{d}]$. So, from (4.4) and (4.5), we have

$$
\begin{aligned}
& \left\|z_{n}-z_{n-1}\right\| \\
& \leq \frac{1}{1-\beta_{n}}\left[\alpha_{n}\left\|f\left(x_{n}\right)-f\left(x_{n-1}\right)\right\|+\gamma_{n}\left(\left\|S_{n} x_{n}-S_{n} x_{n-1}\right\|+\left\|S_{n} x_{n-1}-S_{n-1} x_{n-1}\right\|\right)\right. \\
& +\delta_{n}\left(\left\|x_{n-1}-x_{n}\right\|+M_{0}\left|r_{n-1}-r_{n}\right|\right)+\left|\alpha_{n}-\alpha_{n-1}\right||| f\left(x_{n-1}\right)\left\|+\left|\gamma_{n}-\gamma_{n-1}\right|\right\| S_{n-1} x_{n-1} \| \\
& \left.+\left|\delta_{n}-\delta_{n-1}\right|\left\|J_{r_{n-1}} G x_{n-1}\right\|\right]+\frac{\left|\beta_{n}-\beta_{n-1}\right|}{\left(1-\beta_{n-1}\right)\left(1-\beta_{n}\right)}\left\|y_{n-1}-\beta_{n-1} x_{n-1}\right\| \\
& \leq \frac{1}{1-\beta_{n}}\left[\alpha_{n} \rho\left\|x_{n}-x_{n-1}\right\|+\gamma_{n}\left(\left\|x_{n}-x_{n-1}\right\|+\left\|S_{n} x_{n-1}-S_{n-1} x_{n-1}\right\|\right)\right. \\
& +\delta_{n}\left(\left\|x_{n-1}-x_{n}\right\|+M_{0}\left|r_{n-1}-r_{n}\right|\right)+\left|\alpha_{n}-\alpha_{n-1}\right|\left|f\left(x_{n-1}\right)\left\|+\left|\gamma_{n}-\gamma_{n-1}\right|\right\| S_{n-1} x_{n-1} \|\right. \\
& \left.+\left|\delta_{n}-\delta_{n-1}\right|\left\|J_{r_{n-1}} G x_{n-1}\right\|\right]+\frac{\left|\beta_{n}-\beta_{n-1}\right|}{\left(1-\beta_{n-1}\right)\left(1-\beta_{n}\right)}\left\|y_{n-1}-\beta_{n-1} x_{n-1}\right\| \\
& =\frac{1}{1-\beta_{n}}\left\{\left(1-\beta_{n}-\alpha_{n}(1-\rho)\right)\left\|x_{n}-x_{n-1}\right\|+\gamma_{n}\left\|S_{n} x_{n-1}-S_{n-1} x_{n-1}\right\|+\delta_{n} M_{0}\left|r_{n-1}-r_{n}\right|\right. \\
& \left.+\left|\alpha_{n}-\alpha_{n-1}\right|\left|f\left(x_{n-1}\right)\left\|+\left|\gamma_{n}-\gamma_{n-1}\right||| S_{n-1} x_{n-1}\right\|+\right| \delta_{n}-\delta_{n-1}|| \mid J_{r_{n-1}} G x_{n-1} \|\right\} \\
& +\frac{\left|\beta_{n}-\beta_{n-1}\right|}{\left(1-\beta_{n-1}\right)\left(1-\beta_{n}\right)}\left\|y_{n-1}-\beta_{n-1} x_{n-1}\right\| \\
& =\left(1-\frac{\alpha_{n}(1-\rho)}{1-\beta_{n}}\right)\left\|x_{n}-x_{n-1}\right\|+\frac{\gamma_{n}}{1-\beta_{n}}\left\|S_{n} x_{n-1}-S_{n-1} x_{n-1}\right\|+\frac{\delta_{n} M_{0}}{1-\beta_{n}}\left|r_{n-1}-r_{n}\right| \\
& +\frac{1}{1-\beta_{n}}\left[\left|\alpha_{n}-\alpha_{n-1}\right|\left\|f\left(x_{n-1}\right)\right\|+\left|\gamma_{n}-\gamma_{n-1}\right|\left\|S_{n-1} x_{n-1}\right\|+\left|\delta_{n}-\delta_{n-1}\right|\left\|J_{r_{n-1}} G x_{n-1}\right\|\right] \\
& +\frac{\left|\beta_{n}-\beta_{n-1}\right|}{\left(1-\beta_{n-1}\right)\left(1-\beta_{n}\right)}\left\|y_{n-1}-\beta_{n-1} x_{n-1}\right\| \\
& \leq\left(1-\frac{\alpha_{n}(1-\rho)}{1-\beta_{n}}\right)\left\|x_{n}-x_{n-1}\right\|+\left\|S_{n} x_{n-1}-S_{n-1} x_{n-1}\right\|+M_{0}\left|r_{n-1}-r_{n}\right| \\
& +\frac{1}{1-\beta_{n}}\left[\left|\alpha_{n}-\alpha_{n-1}\right|\left\|f\left(x_{n-1}\right)\right\|+\left|\gamma_{n}-\gamma_{n-1}\right|\left\|S_{n-1} x_{n-1}\right\|+\left|\delta_{n}-\delta_{n-1}\right|\left\|J_{r_{n-1}} G x_{n-1}\right\|\right] \\
& +\frac{\left|\beta_{n}-\beta_{n-1}\right|}{\left(1-\beta_{n-1}\right)\left(1-\beta_{n}\right)}\left\|\alpha_{n-1} f\left(x_{n-1}\right)+\gamma_{n-1} S_{n-1} x_{n-1}+\delta_{n-1} J_{r_{n-1}} G x_{n-1}\right\| \\
& \leq\left(1-\frac{\alpha_{n}(1-\rho)}{1-\beta_{n}}\right)\left\|x_{n}-x_{n-1}\right\|+M_{1}\left[\left|r_{n-1}-r_{n}\right|+\left|\alpha_{n}-\alpha_{n-1}\right|\right.
\end{aligned}
$$




$$
\begin{aligned}
& +\left|\beta_{n}-\beta_{n-1}\right|+\left|\gamma_{n}-\gamma_{n-1}\right| \\
& \left.+\left|\delta_{n}-\delta_{n-1}\right|\right]+\left\|S_{n} x_{n-1}-S_{n-1} x_{n-1}\right\|,
\end{aligned}
$$

where $\sup _{n \geq 0}\left\{\frac{1}{(1-\hat{d})^{2}}\left(\left\|f\left(x_{n}\right)\right\|+\left\|S_{n} x_{n}\right\|+\left\|J_{r_{n}} G x_{n}\right\|+M_{0}\right)\right\} \leq M_{1}$ for some $M_{1}>0$. In the meantime, observe that

$$
x_{n+1}-x_{n}=\sigma_{n}\left(G x_{n}-G x_{n-1}\right)+\left(\sigma_{n}-\sigma_{n-1}\right)\left(G x_{n-1}-z_{n-1}\right)+\left(1-\sigma_{n}\right)\left(z_{n}-z_{n-1}\right) .
$$

This together with (4.6) implies that

$$
\begin{aligned}
\| x_{n+1} & -x_{n} \| \\
\leq & \sigma_{n}\left\|G x_{n}-G x_{n-1}\right\|+\left|\sigma_{n}-\sigma_{n-1}\right|\left\|G x_{n-1}-z_{n-1}\right\|+\left(1-\sigma_{n}\right)\left\|z_{n}-z_{n-1}\right\| \\
\leq & \sigma_{n}\left\|x_{n}-x_{n-1}\right\|+\left|\sigma_{n}-\sigma_{n-1}\right|\left\|G x_{n-1}-z_{n-1}\right\|+\left(1-\sigma_{n}\right)\left\{\left(1-\frac{\alpha_{n}(1-\rho)}{1-\beta_{n}}\right)\left\|x_{n}-x_{n-1}\right\|\right. \\
& +M_{1}\left[\left|r_{n}-r_{n-1}\right|+\left|\alpha_{n}-\alpha_{n-1}\right|+\left|\beta_{n}-\beta_{n-1}\right|+\left|\gamma_{n}-\gamma_{n-1}\right|+\left|\delta_{n}-\delta_{n-1}\right|\right] \\
& \left.+\left\|S_{n} x_{n-1}-S_{n-1} x_{n-1}\right\|\right\} \\
\leq & \left(1-\frac{\left(1-\sigma_{n}\right) \alpha_{n}(1-\rho)}{1-\beta_{n}}\right)\left\|x_{n}-x_{n-1}\right\|+\left|\sigma_{n}-\sigma_{n-1}\right|\left\|G x_{n-1}-z_{n-1}\right\|+M_{1}\left[\left|r_{n}-r_{n-1}\right|\right. \\
& \left.+\left|\alpha_{n}-\alpha_{n-1}\right|+\left|\beta_{n}-\beta_{n-1}\right|+\left|\gamma_{n}-\gamma_{n-1}\right|+\left|\delta_{n}-\delta_{n-1}\right|\right]+\left\|S_{n} x_{n-1}-S_{n-1} x_{n-1}\right\| \\
\leq & \left(1-\frac{\left(1-\sigma_{n}\right) \alpha_{n}(1-\rho)}{1-\beta_{n}}\right)\left\|x_{n}-x_{n-1}\right\|+M_{2}\left[\left|r_{n}-r_{n-1}\right|+\left|\sigma_{n}-\sigma_{n-1}\right|+\left|\alpha_{n}-\alpha_{n-1}\right|\right. \\
& \left.+\left|\beta_{n}-\beta_{n-1}\right|+\left|\gamma_{n}-\gamma_{n-1}\right|+\left|\delta_{n}-\delta_{n-1}\right|\right]+\left\|S_{n} x_{n-1}-S_{n-1} x_{n-1}\right\|,
\end{aligned}
$$

where $\sup _{n \geq 0}\left\{M_{1}+\left\|G x_{n}-z_{n}\right\|\right\} \leq M_{2}$ for some $M_{2}>0$. Since $\frac{\left(1-\sigma_{n}\right) \alpha_{n}(1-\rho)}{1-\beta_{n}} \geq\left(1-\sigma_{n}\right) \alpha_{n}(1-$ $\rho$ ), we obtain from conditions (i) and (v) that $\sum_{n=0}^{\infty} \frac{\left(1-\sigma_{n}\right) \alpha_{n}(1-\rho)}{1-\beta_{n}}=\infty$. Thus, applying Lemma 2.1 to (4.7), we deduce from conditions (iii), (iv) and the assumption on $\left\{S_{n}\right\}$ that

$$
\lim _{n \rightarrow \infty}\left\|x_{n+1}-x_{n}\right\|=0
$$

Next, we show that $\left\|x_{n}-G x_{n}\right\| \rightarrow 0$ as $n \rightarrow \infty$.

Indeed, according to Lemma 2.2, we have from (4.1)

$$
\begin{aligned}
\| y_{n}- & p \|^{2} \\
= & \left\|\alpha_{n}\left(f\left(x_{n}\right)-f(p)\right)+\beta_{n}\left(x_{n}-p\right)+\gamma_{n}\left(S_{n} x_{n}-p\right)+\delta_{n}\left(J_{r_{n}} G x_{n}-p\right)+\alpha_{n}(f(p)-p)\right\|^{2} \\
\leq & \left\|\alpha_{n}\left(f\left(x_{n}\right)-f(p)\right)+\beta_{n}\left(x_{n}-p\right)+\gamma_{n}\left(S_{n} x_{n}-p\right)+\delta_{n}\left(J_{r_{n}} G x_{n}-p\right)\right\|^{2} \\
& +2 \alpha_{n}\left\langle f(p)-p, J\left(y_{n}-p\right)\right\rangle \\
\leq & \alpha_{n}\left\|f\left(x_{n}\right)-f(p)\right\|^{2}+\beta_{n}\left\|x_{n}-p\right\|^{2}+\gamma_{n}\left\|S_{n} x_{n}-p\right\|^{2}+\delta_{n}\left\|J_{r_{n}} G x_{n}-p\right\|^{2} \\
& +2 \alpha_{n}\left\{f(p)-p, J\left(y_{n}-p\right)\right\rangle \\
\leq & \alpha_{n} \rho\left\|x_{n}-p\right\|^{2}+\beta_{n}\left\|x_{n}-p\right\|^{2}+\gamma_{n}\left\|x_{n}-p\right\|^{2}+\delta_{n}\left\|x_{n}-p\right\|^{2}
\end{aligned}
$$




$$
\begin{aligned}
& +2 \alpha_{n}\|f(p)-p\|\left\|y_{n}-p\right\| \\
= & \left(1-\alpha_{n}(1-\rho)\right)\left\|x_{n}-p\right\|^{2}+2 \alpha_{n}\|f(p)-p\|\left\|y_{n}-p\right\| \\
\leq & \left\|x_{n}-p\right\|^{2}+2 \alpha_{n}\|f(p)-p\|\left\|y_{n}-p\right\| .
\end{aligned}
$$

Utilizing Lemma 2.5 , we get from (4.1) and (4.8)

$$
\begin{aligned}
\left\|x_{n+1}-p\right\|^{2}= & \left\|\sigma_{n}\left(G x_{n}-p\right)+\left(1-\sigma_{n}\right)\left(y_{n}-p\right)\right\|^{2} \\
\leq & \sigma_{n}\left\|G x_{n}-p\right\|^{2}+\left(1-\sigma_{n}\right)\left\|y_{n}-p\right\|^{2}-\sigma_{n}\left(1-\sigma_{n}\right) g\left(\left\|G x_{n}-y_{n}\right\|\right) \\
\leq & \sigma_{n}\left\|x_{n}-p\right\|^{2}+\left(1-\sigma_{n}\right)\left[\left\|x_{n}-p\right\|^{2}+2 \alpha_{n}\|f(p)-p\|\left\|y_{n}-p\right\|\right] \\
& -\sigma_{n}\left(1-\sigma_{n}\right) g\left(\left\|G x_{n}-y_{n}\right\|\right) \\
\leq & \left\|x_{n}-p\right\|^{2}+2 \alpha_{n}\|f(p)-p\|\left\|y_{n}-p\right\|-\sigma_{n}\left(1-\sigma_{n}\right) g\left(\left\|G x_{n}-y_{n}\right\|\right),
\end{aligned}
$$

which hence yields

$$
\begin{aligned}
& \sigma_{n}\left(1-\sigma_{n}\right) g\left(\left\|G x_{n}-y_{n}\right\|\right) \\
& \quad \leq\left\|x_{n}-p\right\|^{2}-\left\|x_{n+1}-p\right\|^{2}+2 \alpha_{n}\|f(p)-p\|\left\|y_{n}-p\right\| \\
& \quad \leq\left(\left\|x_{n}-p\right\|+\left\|x_{n+1}-p\right\|\right)\left\|x_{n}-x_{n+1}\right\|+2 \alpha_{n}\|f(p)-p\|\left\|y_{n}-p\right\| .
\end{aligned}
$$

Since $\alpha_{n} \rightarrow 0$ and $\left\|x_{n+1}-x_{n}\right\| \rightarrow 0$, from condition (v) and the boundedness of $\left\{x_{n}\right\}$ and $\left\{y_{n}\right\}$, it follows that

$$
\lim _{n \rightarrow \infty} g\left(\left\|G x_{n}-y_{n}\right\|\right)=0 .
$$

Utilizing the properties of $g$, we have

$$
\lim _{n \rightarrow \infty}\left\|G x_{n}-y_{n}\right\|=0,
$$

which together with (4.1) and (4.3) implies that

$$
\begin{aligned}
\left\|x_{n}-y_{n}\right\| & \leq\left\|x_{n}-x_{n+1}\right\|+\left\|x_{n+1}-y_{n}\right\| \\
& =\left\|x_{n}-x_{n+1}\right\|+\sigma_{n}\left\|G x_{n}-y_{n}\right\| \rightarrow 0 \quad \text { as } n \rightarrow \infty .
\end{aligned}
$$

That is,

$$
\lim _{n \rightarrow \infty}\left\|x_{n}-y_{n}\right\|=0
$$

Since

$$
\left\|x_{n}-G x_{n}\right\| \leq\left\|x_{n}-y_{n}\right\|+\left\|y_{n}-G x_{n}\right\|,
$$

it immediately follows from (4.9) and (4.10) that

$$
\lim _{n \rightarrow \infty}\left\|x_{n}-G x_{n}\right\|=0
$$


On the other hand, observe that $y_{n}$ can be rewritten as follows:

$$
\begin{aligned}
y_{n} & =\alpha_{n} f\left(x_{n}\right)+\beta_{n} x_{n}+\gamma_{n} S_{n} x_{n}+\delta_{n} J_{r_{n}} G x_{n} \\
& =\alpha_{n} f\left(x_{n}\right)+\beta_{n} x_{n}+\left(\gamma_{n}+\delta_{n}\right) \frac{\gamma_{n} S_{n} x_{n}+\delta_{n} J_{r_{n}} G x_{n}}{\gamma_{n}+\delta_{n}} \\
& =\alpha_{n} f\left(x_{n}\right)+\beta_{n} x_{n}+e_{n} \hat{z}_{n},
\end{aligned}
$$

where $e_{n}=\gamma_{n}+\delta_{n}$ and $\hat{z}_{n}=\frac{\gamma_{n} S_{n} x_{n}+\delta_{n} J_{r_{n}} G x_{n}}{\gamma_{n}+\delta_{n}}$. Utilizing Lemma 2.11, we have

$$
\begin{aligned}
\left\|y_{n}-p\right\|^{2}= & \left\|\alpha_{n}\left(f\left(x_{n}\right)-p\right)+\beta_{n}\left(x_{n}-p\right)+e_{n}\left(\hat{z}_{n}-p\right)\right\|^{2} \\
\leq & \alpha_{n}\left\|f\left(x_{n}\right)-p\right\|^{2}+\beta_{n}\left\|x_{n}-p\right\|^{2}+e_{n}\left\|\hat{z}_{n}-p\right\|^{2}-\beta_{n} e_{n} g_{1}\left(\left\|\hat{z}_{n}-x_{n}\right\|\right) \\
= & \alpha_{n}\left\|f\left(x_{n}\right)-p\right\|^{2}+\beta_{n}\left\|x_{n}-p\right\|^{2}-\beta_{n} e_{n} g_{1}\left(\left\|\hat{z}_{n}-x_{n}\right\|\right) \\
& +e_{n}\left\|\frac{\gamma_{n} S_{n} x_{n}+\delta_{n} J_{r_{n}} G x_{n}}{\gamma_{n}+\delta_{n}}-p\right\|^{2} \\
= & \alpha_{n}\left\|f\left(x_{n}\right)-p\right\|^{2}+\beta_{n}\left\|x_{n}-p\right\|^{2}-\beta_{n} e_{n} g_{1}\left(\left\|\hat{z}_{n}-x_{n}\right\|\right) \\
& +e_{n}\left\|\frac{\gamma_{n}}{\gamma_{n}+\delta_{n}}\left(S_{n} x_{n}-p\right)+\frac{\delta_{n}}{\gamma_{n}+\delta_{n}}\left(J_{r_{n}} G x_{n}-p\right)\right\|^{2} \\
\leq & \alpha_{n}\left\|f\left(x_{n}\right)-p\right\|^{2}+\beta_{n}\left\|x_{n}-p\right\|^{2}-\beta_{n} e_{n} g_{1}\left(\left\|\hat{z}_{n}-x_{n}\right\|\right) \\
& +e_{n}\left[\frac{\gamma_{n}}{\gamma_{n}+\delta_{n}}\left\|S_{n} x_{n}-p\right\|^{2}+\frac{\delta_{n}}{\gamma_{n}+\delta_{n}}\left\|J_{r_{n}} G x_{n}-p\right\|^{2}\right] \\
\leq & \alpha_{n}\left\|f\left(x_{n}\right)-p\right\|^{2}+\beta_{n}\left\|x_{n}-p\right\|^{2}-\beta_{n} e_{n} g_{1}\left(\left\|\hat{z}_{n}-x_{n}\right\|\right) \\
& +e_{n}\left[\frac{\gamma_{n}}{\gamma_{n}+\delta_{n}}\left\|x_{n}-p\right\|^{2}+\frac{\delta_{n}}{\gamma_{n}+\delta_{n}}\left\|x_{n}-p\right\|^{2}\right] \\
= & \alpha_{n}\left\|f\left(x_{n}\right)-p\right\|^{2}+\left(1-\alpha_{n}\right)\left\|x_{n}-p\right\|^{2}-\beta_{n} e_{n} g_{1}\left(\left\|\hat{z}_{n}-x_{n}\right\|\right) \\
\leq & \alpha_{n}\left\|f\left(x_{n}\right)-p\right\|^{2}+\left\|x_{n}-p\right\|^{2}-\beta_{n} e_{n} g_{1}\left(\left\|\hat{z}_{n}-x_{n}\right\|\right) \\
&
\end{aligned}
$$

which hence implies that

$$
\begin{aligned}
\beta_{n} e_{n} g_{1}\left(\left\|\hat{z}_{n}-x_{n}\right\|\right) & \leq \alpha_{n}\left\|f\left(x_{n}\right)-p\right\|^{2}+\left\|x_{n}-p\right\|^{2}-\left\|y_{n}-p\right\|^{2} \\
& \leq \alpha_{n}\left\|f\left(x_{n}\right)-p\right\|^{2}+\left(\left\|x_{n}-p\right\|+\left\|y_{n}-p\right\|\right)\left\|x_{n}-y_{n}\right\| .
\end{aligned}
$$

Utilizing (4.10), conditions (i), (ii), (v) and the boundedness of $\left\{x_{n}\right\},\left\{y_{n}\right\}$ and $\left\{f\left(x_{n}\right)\right\}$, we get

$$
\lim _{n \rightarrow \infty} g_{1}\left(\left\|\hat{z}_{n}-x_{n}\right\|\right)=0
$$

From the properties of $g_{1}$, we have

$$
\lim _{n \rightarrow \infty}\left\|\hat{z}_{n}-x_{n}\right\|=0
$$


Utilizing Lemma 2.5 and the definition of $\hat{z}_{n}$, we have

$$
\begin{aligned}
\left\|\hat{z}_{n}-p\right\|^{2}= & \left\|\frac{\gamma_{n} S_{n} x_{n}+\delta_{n} J_{r_{n}} G x_{n}}{\gamma_{n}+\delta_{n}}-p\right\|^{2} \\
= & \left\|\frac{\gamma_{n}}{\gamma_{n}+\delta_{n}}\left(S_{n} x_{n}-p\right)+\frac{\delta_{n}}{\gamma_{n}+\delta_{n}}\left(J_{r_{n}} G x_{n}-p\right)\right\|^{2} \\
\leq & \frac{\gamma_{n}}{\gamma_{n}+\delta_{n}}\left\|S_{n} x_{n}-p\right\|^{2}+\frac{\delta_{n}}{\gamma_{n}+\delta_{n}}\left\|J_{r_{n}} G x_{n}-p\right\|^{2} \\
& -\frac{\gamma_{n} \delta_{n}}{\left(\gamma_{n}+\delta_{n}\right)^{2}} g_{2}\left(\left\|J_{r_{n}} G x_{n}-S_{n} x_{n}\right\|\right) \\
\leq & \left\|x_{n}-p\right\|^{2}-\frac{\gamma_{n} \delta_{n}}{\left(\gamma_{n}+\delta_{n}\right)^{2}} g_{2}\left(\left\|J_{r_{n}} G x_{n}-S_{n} x_{n}\right\|\right),
\end{aligned}
$$

which leads to

$$
\begin{aligned}
\frac{\gamma_{n} \delta_{n}}{\left(\gamma_{n}+\delta_{n}\right)^{2}} g_{2}\left(\left\|J_{r_{n}} G x_{n}-S_{n} x_{n}\right\|\right) & \leq\left\|x_{n}-p\right\|^{2}-\left\|\hat{z}_{n}-p\right\|^{2} \\
& \leq\left(\left\|x_{n}-p\right\|+\left\|\hat{z}_{n}-p\right\|\right)\left\|x_{n}-\hat{z}_{n}\right\| .
\end{aligned}
$$

Since $\left\{x_{n}\right\}$ and $\left\{\hat{z}_{n}\right\}$ are bounded, we deduce from (4.12) and condition (ii) that

$$
\lim _{n \rightarrow \infty} g_{2}\left(\left\|S_{n} x_{n}-J_{r_{n}} G x_{n}\right\|\right)=0
$$

From the properties of $g_{2}$, we have

$$
\lim _{n \rightarrow \infty}\left\|S_{n} x_{n}-J_{r_{n}} G x_{n}\right\|=0 .
$$

Furthermore, $y_{n}$ can also be rewritten as follows:

$$
\begin{aligned}
y_{n} & =\alpha_{n} f\left(x_{n}\right)+\beta_{n} x_{n}+\gamma_{n} S_{n} x_{n}+\delta_{n} J_{r_{n}} G x_{n} \\
& =\beta_{n} x_{n}+\gamma_{n} S_{n} x_{n}+\left(\alpha_{n}+\delta_{n}\right) \frac{\alpha_{n} f\left(x_{n}\right)+\delta_{n} J_{r_{n}} G x_{n}}{\alpha_{n}+\delta_{n}} \\
& =\beta_{n} x_{n}+\gamma_{n} S_{n} x_{n}+d_{n} \tilde{z}_{n},
\end{aligned}
$$

where $d_{n}=\alpha_{n}+\delta_{n}$ and $\tilde{z}_{n}=\frac{\alpha_{n} f\left(x_{n}\right)+\delta_{n} J_{r_{n}} G x_{n}}{\alpha_{n}+\delta_{n}}$. Utilizing Lemma 2.11 and the convexity of $\|\cdot\|^{2}$, we have

$$
\begin{aligned}
& \left\|y_{n}-p\right\|^{2} \\
& =\left\|\beta_{n}\left(x_{n}-p\right)+\gamma_{n}\left(S_{n} x_{n}-p\right)+d_{n}\left(\tilde{z}_{n}-p\right)\right\|^{2} \\
& \leq \beta_{n}\left\|x_{n}-p\right\|^{2}+\gamma_{n}\left\|S_{n} x_{n}-p\right\|^{2}+d_{n}\left\|\tilde{z}_{n}-p\right\|^{2}-\beta_{n} \gamma_{n} g_{3}\left(\left\|x_{n}-S_{n} x_{n}\right\|\right) \\
& =\beta_{n}\left\|x_{n}-p\right\|^{2}+\gamma_{n}\left\|S_{n} x_{n}-p\right\|^{2}+d_{n}\left\|\frac{\alpha_{n} f\left(x_{n}\right)+\delta_{n} J_{r_{n}} G x_{n}}{\alpha_{n}+\delta_{n}}-p\right\|^{2} \\
& \quad-\beta_{n} \gamma_{n} g_{3}\left(\left\|x_{n}-S_{n} x_{n}\right\|\right)
\end{aligned}
$$




$$
\begin{aligned}
= & \beta_{n}\left\|x_{n}-p\right\|^{2}+\gamma_{n}\left\|S_{n} x_{n}-p\right\|^{2}+d_{n}\left\|\frac{\alpha_{n}}{\alpha_{n}+\delta_{n}}\left(f\left(x_{n}\right)-p\right)+\frac{\delta_{n}}{\alpha_{n}+\delta_{n}}\left(J_{n} G x_{n}-p\right)\right\|^{2} \\
& -\beta_{n} \gamma_{n} g_{3}\left(\left\|x_{n}-S_{n} x_{n}\right\|\right) \\
\leq & \beta_{n}\left\|x_{n}-p\right\|^{2}+\gamma_{n}\left\|x_{n}-p\right\|^{2}+d_{n}\left[\frac{\alpha_{n}}{\alpha_{n}+\delta_{n}}\left\|f\left(x_{n}\right)-p\right\|^{2}+\frac{\delta_{n}}{\alpha_{n}+\delta_{n}}\left\|J_{r_{n}} G x_{n}-p\right\|^{2}\right] \\
& -\beta_{n} \gamma_{n} g_{3}\left(\left\|x_{n}-S_{n} x_{n}\right\|\right) \\
\leq & \alpha_{n}\left\|f\left(x_{n}\right)-p\right\|^{2}+\left(\beta_{n}+\gamma_{n}\right)\left\|x_{n}-p\right\|^{2}+\delta_{n}\left\|x_{n}-p\right\|^{2}-\beta_{n} \gamma_{n} g_{3}\left(\left\|x_{n}-S_{n} x_{n}\right\|\right) \\
= & \alpha_{n}\left\|f\left(x_{n}\right)-p\right\|^{2}+\left(1-\alpha_{n}\right)\left\|x_{n}-p\right\|^{2}-\beta_{n} \gamma_{n} g_{3}\left(\left\|x_{n}-S_{n} x_{n}\right\|\right) \\
\leq & \alpha_{n}\left\|f\left(x_{n}\right)-p\right\|^{2}+\left\|x_{n}-p\right\|^{2}-\beta_{n} \gamma_{n} g_{3}\left(\left\|x_{n}-S_{n} x_{n}\right\|\right),
\end{aligned}
$$

which hence implies that

$$
\begin{aligned}
\beta_{n} \gamma_{n} g_{3}\left(\left\|x_{n}-S_{n} x_{n}\right\|\right) & \leq \alpha_{n}\left\|f\left(x_{n}\right)-p\right\|^{2}+\left\|x_{n}-p\right\|^{2}-\left\|y_{n}-p\right\|^{2} \\
& \leq \alpha_{n}\left\|f\left(x_{n}\right)-p\right\|^{2}+\left(\left\|x_{n}-p\right\|+\left\|y_{n}-p\right\|\right)\left\|x_{n}-y_{n}\right\| .
\end{aligned}
$$

Utilizing (4.10), conditions (i), (ii), (v) and the boundedness of $\left\{x_{n}\right\},\left\{y_{n}\right\}$ and $\left\{f\left(x_{n}\right)\right\}$, we get

$$
\lim _{n \rightarrow \infty} g_{3}\left(\left\|x_{n}-S_{n} x_{n}\right\|\right)=0
$$

From the properties of $g_{3}$, we have

$$
\lim _{n \rightarrow \infty}\left\|x_{n}-S_{n} x_{n}\right\|=0
$$

Thus, from (4.13) and (4.14), we get

$$
\left\|x_{n}-J_{r_{n}} G x_{n}\right\| \leq\left\|x_{n}-S_{n} x_{n}\right\|+\left\|S_{n} x_{n}-J_{r_{n}} G x_{n}\right\| \rightarrow 0 \quad \text { as } n \rightarrow \infty \text {. }
$$

That is,

$$
\lim _{n \rightarrow \infty}\left\|x_{n}-J_{r_{n}} G x_{n}\right\|=0
$$

In terms of (4.14) and Lemma 2.6, we have

$$
\left\|x_{n}-S x_{n}\right\| \leq\left\|x_{n}-S_{n} x_{n}\right\|+\left\|S_{n} x_{n}-S x_{n}\right\| \rightarrow 0 \quad \text { as } n \rightarrow \infty .
$$

That is,

$$
\lim _{n \rightarrow \infty}\left\|x_{n}-S x_{n}\right\|=0
$$

Furthermore, repeating the same arguments as those of (3.30) in the proof of Theorem 3.1, we can conclude that

$$
\lim _{n \rightarrow \infty}\left\|x_{n}-J_{r} x_{n}\right\|=0
$$

for a fixed number $r$ such that $\varepsilon>r>0$. 
Define a mapping $W x=\left(1-\theta_{1}-\theta_{2}\right) J_{r} x+\theta_{1} S x+\theta_{2} G x$, where $\theta_{1}, \theta_{2} \in(0,1)$ are two constants with $\theta_{1}+\theta_{2}<1$. Then by Lemma 2.8, we have that $\operatorname{Fix}(W)=\operatorname{Fix}\left(J_{r}\right) \cap \operatorname{Fix}(S) \cap \operatorname{Fix}(G)=F$. We observe that

$$
\begin{aligned}
\left\|x_{n}-W x_{n}\right\| & =\left\|\left(1-\theta_{1}-\theta_{2}\right)\left(x_{n}-J_{r} x_{n}\right)+\theta_{1}\left(x_{n}-S x_{n}\right)+\theta_{2}\left(x_{n}-G x_{n}\right)\right\| \\
& \leq\left(1-\theta_{1}-\theta_{2}\right)\left\|x_{n}-J_{r} x_{n}\right\|+\theta_{1}\left\|x_{n}-S x_{n}\right\|+\theta_{2}\left\|x_{n}-G x_{n}\right\| .
\end{aligned}
$$

From (4.11), (4.16) and (4.17), we obtain

$$
\lim _{n \rightarrow \infty}\left\|x_{n}-W x_{n}\right\|=0
$$

Now, we claim that

$$
\limsup _{n \rightarrow \infty}\left\langle f(q)-q, J\left(x_{n}-q\right)\right\rangle \leq 0
$$

where $q=s-\lim _{t \rightarrow 0} x_{t}$ with $x_{t}$ being the fixed point of the contraction

$$
x \mapsto t f(x)+(1-t) W x .
$$

Then $x_{t}$ solves the fixed point equation $x_{t}=t f\left(x_{t}\right)+(1-t) W x_{t}$. Repeating the same arguments as those of (3.37) in the proof of Theorem 3.1, we can obtain that

$$
\begin{aligned}
\limsup _{n \rightarrow \infty}\left\langle f(q)-q, J\left(x_{n}-q\right)\right\rangle & =\limsup _{t \rightarrow 0} \limsup _{n \rightarrow \infty}\left\langle f(q)-q, J\left(x_{n}-q\right)\right\rangle \\
& \leq \limsup _{t \rightarrow 0} \limsup _{n \rightarrow \infty}\left\langle f(q)-q, J\left(x_{n}-q\right)-J\left(x_{n}-x_{t}\right)\right\rangle .
\end{aligned}
$$

Since $X$ has a uniformly Gâteaux differentiable norm, the duality mapping $J$ is norm-toweak* uniformly continuous on bounded subsets of $X$. Consequently, the two limits are interchangeable, and hence (4.19) holds. From (4.10), we get $\left(y_{n}-q\right)-\left(x_{n}-q\right) \rightarrow 0$. Noticing the norm-to-weak* uniform continuity of $J$ on bounded subsets of $X$, we deduce from (4.19) that

$$
\begin{aligned}
& \limsup _{n \rightarrow \infty}\left\langle f(q)-q, J\left(y_{n}-q\right)\right\rangle \\
& \quad=\limsup _{n \rightarrow \infty}\left(\left\langle f(q)-q, J\left(x_{n}-q\right)\right\rangle+\left\langle f(q)-q, J\left(y_{n}-q\right)-J\left(x_{n}-q\right)\right\rangle\right) \\
& \quad=\limsup _{n \rightarrow \infty}\left\langle f(q)-q, J\left(x_{n}-q\right)\right\rangle \leq 0 .
\end{aligned}
$$

Finally, let us show that $x_{n} \rightarrow q$ as $n \rightarrow \infty$. Indeed, observe that

$$
\begin{aligned}
&\left\|y_{n}-q\right\|^{2} \\
&=\left\|\alpha_{n}\left(f\left(x_{n}\right)-f(q)\right)+\beta_{n}\left(x_{n}-q\right)+\gamma_{n}\left(S_{n} x_{n}-q\right)+\delta_{n}\left(J_{r_{n}} G x_{n}-q\right)+\alpha_{n}(f(q)-q)\right\|^{2} \\
& \leq\left\|\alpha_{n}\left(f\left(x_{n}\right)-f(q)\right)+\beta_{n}\left(x_{n}-q\right)+\gamma_{n}\left(S_{n} x_{n}-q\right)+\delta_{n}\left(J_{r_{n}} G x_{n}-q\right)\right\|^{2} \\
&+2 \alpha_{n}\left\langle f(q)-q, J\left(y_{n}-q\right)\right\rangle
\end{aligned}
$$




$$
\begin{aligned}
\leq & \alpha_{n}\left\|f\left(x_{n}\right)-f(q)\right\|^{2}+\beta_{n}\left\|x_{n}-q\right\|^{2}+\gamma_{n}\left\|S_{n} x_{n}-q\right\|^{2}+\delta_{n}\left\|J_{r_{n}} G x_{n}-q\right\|^{2} \\
& +2 \alpha_{n}\left\langle f(q)-q, J\left(y_{n}-q\right)\right\rangle \\
\leq & \alpha_{n} \rho\left\|x_{n}-q\right\|^{2}+\beta_{n}\left\|x_{n}-q\right\|^{2}+\gamma_{n}\left\|x_{n}-q\right\|+\delta_{n}\left\|x_{n}-q\right\|^{2}+2 \alpha_{n}\left\langle f(q)-q, J\left(y_{n}-q\right)\right\rangle \\
= & \left(\alpha_{n} \rho+\beta_{n}+\gamma_{n}+\delta_{n}\right)\left\|x_{n}-q\right\|^{2}+2 \alpha_{n}\left\langle f(q)-q, J\left(y_{n}-q\right)\right\rangle \\
= & \left(1-\alpha_{n}(1-\rho)\right)\left\|x_{n}-q\right\|^{2}+2 \alpha_{n}\left\langle f(q)-q, J\left(y_{n}-q\right)\right\rangle,
\end{aligned}
$$

and hence

$$
\begin{aligned}
\left\|x_{n+1}-q\right\|^{2} \\
\quad \leq \sigma_{n}\left\|G x_{n}-q\right\|^{2}+\left(1-\sigma_{n}\right)\left\|y_{n}-q\right\|^{2} \\
\quad \leq \sigma_{n}\left\|x_{n}-q\right\|^{2}+\left(1-\sigma_{n}\right)\left[\left(1-\alpha_{n}(1-\rho)\right)\left\|x_{n}-q\right\|^{2}+2 \alpha_{n}\left\langle f(q)-q, J\left(y_{n}-q\right)\right\rangle\right] \\
\quad=\left(1-\left(1-\sigma_{n}\right) \alpha_{n}(1-\rho)\right)\left\|x_{n}-q\right\|^{2}+2\left(1-\sigma_{n}\right) \alpha_{n}\left\langle f(q)-q, J\left(y_{n}-q\right)\right\rangle \\
\quad=\left(1-\left(1-\sigma_{n}\right) \alpha_{n}(1-\rho)\right)\left\|x_{n}-q\right\|^{2}+\left(1-\sigma_{n}\right) \alpha_{n}(1-\rho) \frac{2\left\langle f(q)-q, J\left(y_{n}-q\right)\right\rangle}{1-\rho} .
\end{aligned}
$$

Applying Lemma 2.1 to (4.21), we conclude from conditions (i), (v) and (4.20) that $x_{n} \rightarrow q$ as $n \rightarrow \infty$. This completes the proof.

Corollary 4.1 Let $C$ be a nonempty closed convex subset of a uniformly convex Banach space $X$, which has a uniformly Gâteaux differentiable norm. Let $\Pi_{C}$ be a sunny nonexpansive retraction from $X$ onto $C$. Let $A \subset X \times X$ be an accretive operator in $X$ such that $\overline{D(A)} \subset C \subset \bigcap_{r>0} R(I+r A)$. Let $V: C \rightarrow C$ be a self-mapping such that $I-V: C \rightarrow X$ is $\lambda$-strictly pseudocontractive and $\alpha$-strongly accretive with $\alpha+\lambda \geq 1$. Let $f: C \rightarrow C$ be a contraction with coefficient $\rho \in(0,1)$. Let $\left\{S_{i}\right\}_{i=0}^{\infty}$ be a countable family of nonexpansive mappings of $C$ into itself such that $F=\bigcap_{i=0}^{\infty} \operatorname{Fix}\left(S_{i}\right) \cap \operatorname{Fix}(V) \cap A^{-1} 0 \neq \emptyset$. For arbitrarily given $x_{0} \in C$, let $\left\{x_{n}\right\}$ be the sequence generated by

$$
\left\{\begin{array}{l}
y_{n}=\alpha_{n} f\left(x_{n}\right)+\beta_{n} x_{n}+\gamma_{n} S_{n} x_{n}+\delta_{n} J_{r_{n}}((1-l) I+l V) x_{n}, \\
x_{n+1}=\sigma_{n}((1-l) I+l V) x_{n}+\left(1-\sigma_{n}\right) y_{n}, \quad \forall n \geq 0
\end{array}\right.
$$

where $1-\frac{\lambda}{1+\lambda}\left(1-\sqrt{\frac{1-\alpha}{\lambda}}\right) \leq l \leq 1$, and $\left\{\alpha_{n}\right\},\left\{\beta_{n}\right\},\left\{\gamma_{n}\right\},\left\{\delta_{n}\right\}$ and $\left\{\sigma_{n}\right\}$ are the sequences in $(0,1)$ such that $\alpha_{n}+\beta_{n}+\gamma_{n}+\delta_{n}=1$ for all $n \geq 0$. Suppose that the following conditions hold:

(i) $\lim _{n \rightarrow \infty} \alpha_{n}=0$ and $\sum_{n=0}^{\infty} \alpha_{n}=\infty$;

(ii) $\left\{\gamma_{n}\right\},\left\{\delta_{n}\right\} \subset[c, d]$ for some $c, d \in(0,1)$;

(iii) $\sum_{n=1}^{\infty}\left(\left|\sigma_{n}-\sigma_{n-1}\right|+\left|\alpha_{n}-\alpha_{n-1}\right|+\left|\beta_{n}-\beta_{n-1}\right|+\left|\gamma_{n}-\gamma_{n-1}\right|+\left|\delta_{n}-\delta_{n-1}\right|\right)<\infty$;

(iv) $\sum_{n=1}^{\infty}\left|r_{n}-r_{n-1}\right|<\infty$ and $r_{n} \geq \varepsilon>0$ for all $n \geq 0$;

(v) $0<\liminf _{n \rightarrow \infty} \beta_{n} \leq \limsup \sup _{n \rightarrow \infty} \beta_{n}<1$ and $0<\liminf _{n \rightarrow \infty} \sigma_{n} \leq \lim \sup _{n \rightarrow \infty} \sigma_{n}<1$. Assume that $\sum_{n=1}^{\infty} \sup _{x \in D}\left\|S_{n} x-S_{n-1} x\right\|<\infty$ for any bounded subset $D$ of $C$, and let $S$ be a mapping of $C$ into itself defined by $S x=\lim _{n \rightarrow \infty} S_{n} x$ for all $x \in C$, and suppose that $\operatorname{Fix}(S)=$ $\bigcap_{i=0}^{\infty} \operatorname{Fix}\left(S_{i}\right)$. Then $\left\{x_{n}\right\}$ converges strongly to $q \in F$, which solves the following VIP:

$$
\langle q-f(q), J(q-p)\rangle \leq 0, \quad \forall p \in F .
$$


Proof In Theorem 4.1, we put $B_{1}=I-V, B_{2}=0$ and $\mu_{1}=l$, where $1-\frac{\lambda}{1+\lambda}\left(1-\sqrt{\frac{1-\alpha}{\lambda}}\right) \leq l \leq 1$. Then GSVI (1.1) is equivalent to the VIP of finding $x^{*} \in C$ such that

$$
\left\langle B_{1} x^{*}, J\left(x-x^{*}\right)\right| \geq 0, \quad \forall x \in C .
$$

In this case, $B_{1}: C \rightarrow X$ is $\lambda$-strictly pseudocontractive and $\alpha$-strongly accretive. Repeating the same arguments as those in the proof of Corollary 3.1, we can infer that $\operatorname{Fix}(V)=$ $\operatorname{VI}\left(C, B_{1}\right)$. Accordingly, $F=\bigcap_{i=0}^{\infty} \operatorname{Fix}\left(S_{i}\right) \cap \Omega \cap A^{-1} 0=\bigcap_{i=0}^{\infty} \operatorname{Fix}\left(S_{i}\right) \cap \operatorname{Fix}(V) \cap A^{-1} 0$, and

$$
G x_{n}=((1-l) I+l V) x_{n}, \quad \forall n \geq 0 .
$$

So, the scheme (4.1) reduces to (4.22). Therefore, the desired result follows from Theorem 4.1.

Remark 4.1 Theorem 4.1 improves, extends, supplements and develops Jung [22, Theorem 3.1], Ceng and Yao [2, Theorem 3.1] and Cai and $\mathrm{Bu}$ [3, Theorem 3.1] in the following aspects.

(i) The problem of finding a point $q \in \bigcap_{i=0}^{\infty} \operatorname{Fix}\left(S_{i}\right) \cap \Omega \cap A^{-1} 0$ in our Theorem 4.1 is more general and more subtle than any of the problems of finding a point $q \in A^{-1} 0$ in [22, Theorem 3.1], the problem of finding a point $q \in \operatorname{Fix}(T)$ in [2, Theorem 3.1], and the problem of finding a point $q \in \bigcap_{i=1}^{\infty} \operatorname{Fix}\left(T_{i}\right) \cap \Omega$ in [3, Theorem 3.1].

(ii) The iterative scheme in [22, Theorem 3.1] is extended to develop the iterative scheme (4.1) of Theorem 4.1 by virtue of the iterative schemes of [2, Theorems 3.1] and [3, Theorem 3.1]. The iterative scheme (4.1) of Theorem 4.1 is more advantageous and more flexible than the iterative scheme of [2, Theorem 3.1], because it can be applied to solving three problems (i.e., GSVI (1.1), fixed point problem and zero point problem) and involves several parameter sequences $\left\{\sigma_{n}\right\},\left\{\alpha_{n}\right\},\left\{\beta_{n}\right\},\left\{\gamma_{n}\right\},\left\{\delta_{n}\right\}$ and $\left\{r_{n}\right\}$.

(iii) Theorem 4.1 extends and generalizes Ceng and Yao [2, Theorem 3.1] from a nonexpansive mapping to a countable family of nonexpansive mappings, and Jung [22, Theorem 3.1] to the setting of a countable family of nonexpansive mappings and GSVI (1.1) for two strictly pseudocontractive and strongly accretive mappings. In the meantime, Theorem 4.1 extends and generalizes $\mathrm{Cai}$ and $\mathrm{Bu}$ [3, Theorem 3.1] to the setting of an accretive operator.

(iv) The iterative scheme (4.1) in Theorem 4.1 is very different from any in [22, Theorem 3.1], [2, Theorem 3.1] and [3, Theorem 3.1] because the mapping $J_{r_{n}}$ in [22, Theorem 3.1] and the mapping $G$ in [3, Theorem 3.1] are replaced by the same composite mapping $J_{r_{n}} G$ in the iterative scheme (4.1) of Theorem 4.1.

(v) Cai and Bu's proof in [3, Theorem 3.1] depends on the argument techniques in [6], the inequality in 2-uniformly smooth Banach spaces (see Proposition 2.1) and the inequality in smooth and uniform convex Banach spaces (see Proposition 2.2). However, the proof of Theorem 4.1 does not depend on the argument techniques in [6], the inequality in 2uniformly smooth Banach spaces (see Proposition 2.1), and the inequality in smooth and uniform convex Banach spaces (see Proposition 2.2). It depends on only the inequalities in uniform convex Banach spaces (see Lemmas 2.5 and 2.11 in Section 2 of this paper) and the resolvent identity for accretive operators (see Proposition 1.1). 
(vi) The assumption of the uniformly convex and 2-uniformly smooth Banach space $X$ in [3, Theorem 3.1] is weakened to the one of the uniformly convex Banach space $X$ having a uniformly Gâteaux differentiable norm in Theorem 4.1. Moreover, the assumption of the uniformly smooth Banach space $X$ in [2, Theorem 3.1] is replaced by the one of the uniformly convex Banach space $X$ having a uniformly Gâteaux differentiable norm in Theorem 4.1. It is worth emphasizing that the assumption of asymptotic regularity on $\left\{x_{n}\right\}$ in [22, Theorem 3.1] is dropped by Theorem 4.1.

\section{Competing interests}

The authors declare that they have no competing interests.

\section{Authors' contributions}

All authors contributed equally and significantly in writing this paper. All authors read and approved the final manuscript.

\section{Author details}

${ }^{1}$ Department of Mathematics, Shanghai Normal University, and Scientific Computing Key Laboratory of Shanghai Universities, Shanghai, 200234, China. ${ }^{2}$ Center for Fundamental Science, Kaohsiung Medical University, Kaohsiung, 807 Taiwan.

\section{Acknowledgements}

The first author was partially supported by the National Science Foundation of China (11071169), the Innovation Program of Shanghai Municipal Education Commission (09ZZ133) and the Ph.D. Program Foundation of Ministry of Education of China (20123127110002). The second author was partially supported by a grant from the NSC 102-2115-M-037-001.

Received: 17 June 2013 Accepted: 6 September 2013 Published: 07 Nov 2013

\section{References}

1. Yao, Y, Chen, R, Yao, JC: Strong convergence and certain control conditions for modified Mann iteration. Nonlinear Anal. 68, 1687-1693 (2008)

2. Ceng, LC, Yao, JC: Convergence and certain control conditions for hybrid viscosity approximation methods. Nonlinear Anal. 73(7), 2078-2087 (2010)

3. Cai, G, Bu, SQ: Convergence analysis for variational inequality problems and fixed point problems in 2-uniformly smooth and uniformly convex Banach spaces. Math. Comput. Model. 55, 538-546 (2012)

4. Verma, RU: On a new system of nonlinear variational inequalities and associated iterative algorithms. Math. Sci. Res. Hot-Line 3(8), 65-68 (1999)

5. Lions, JL, Stampacchia, G: Variational inequalities. Commun. Pure Appl. Math. 20, 493-512 (1967)

6. Ceng, LC, Wang, CY, Yao, JC: Strong convergence theorems by a relaxed extragradient method for a general system of variational inequalities. Math. Methods Oper. Res. 67, 375-390 (2008)

7. Korpelevich, GM: An extragradient method for finding saddle points and for other problems. Èkon. Mat. Metody 12 747-756 (1976)

8. Yao, Y, Liou, YC, Kang, SM, Yu, YL: Algorithms with strong convergence for a system of nonlinear variational inequalities in Banach spaces. Nonlinear Anal. 74(17), 6024-6034 (2011)

9. liduka, H, Takahashi, W: Strong convergence theorems for nonexpansive mappings and inverse-strongly monotone mappings. Nonlinear Anal. 61, 341-350 (2005)

10. Ceng, LC, Ansari, QH, Yao, JC: Relaxed extragradient iterative methods for variational inequalities. Appl. Math. Comput. 218, 1112-1123(2011)

11. Ceng, LC, Ansari, QH, Wong, NC, Yao, JC: An extragradient-like approximation method for variational inequalities and fixed point problems. Fixed Point Theory Appl. 2011, Article ID 22 (2011)

12. Ceng, LC, Hadjisavvas, N, Wong, NC: Strong convergence theorem by a hybrid extragradient-like approximation method for variational inequalities and fixed point problems. J. Glob. Optim. 46, 635-646 (2010)

13. Ceng, LC, Guu, SM, Yao, JC: Finding common solutions of a variational inequality, a general system of variationa inequalities, and a fixed-point problem via a hybrid extragradient method. Fixed Point Theory Appl. 2011, Article ID $626159(2011)$

14. Zeng, LC, Yao, JC: Strong convergence theorem by an extragradient method for fixed point problems and variational inequality problems. Taiwan. J. Math. 10, 1293-1303 (2006)

15. Aoyama, K, liduka, H, Takahashi, W: Weak convergence of an iterative sequence for accretive operators in Banach spaces. Fixed Point Theory Appl. 2006, Article ID 35390 (2006)

16. Ceng, LC, Petrusel, A: Krasnoselski-Mann iterations for hierarchical fixed point problems for a finite family of nonself mappings in Banach spaces. J. Optim. Theory Appl. 146, 617-639 (2010)

17. Xu, HK: Viscosity approximation methods for nonexpansive mappings. J. Math. Anal. Appl. 298, $279-291$ (2004)

18. Ceng, LC, Xu, HK, Yao, JC: Strong convergence of an iterative method with perturbed mappings for nonexpansive and accretive operators. Numer. Funct. Anal. Optim. 29(3-4), 324-345 (2008)

19. Barbu, V: Nonlinear Semigroups and Differential Equations in Banach Spaces. Noordhoff, Leiden (1976)

20. Aoyama, K, Kimura, Y, Takahashi, W, Toyoda, T: Approximation of common fixed points of a countable family of nonexpansive mappings in Banach spaces. Nonlinear Anal. 67, 2350-2360 (2007) 
21. Ceng, LC, Khan, AR, Ansari, QH, Yao, JC: Strong convergence of composite iterative schemes for zeros of $m$-accretive operators in Banach space. Nonlinear Anal. 70, 1830-1840 (2009)

22. Jung, JS: Convergence of composite iterative methods for finding zeros of accretive operators. Nonlinear Anal. 71 1736-1746 (2009)

23. Takahashi, Y, Hashimoto, K, Kato, M: On sharp uniform convexity, smoothness, and strong type, cotype inequalities. J. Nonlinear Convex Anal. 3, 267-281 (2002)

24. Zeng, LC, Lee, GM, Wong, NC: Ishikawa iteration with errors for approximating fixed points of strictly pseudocontractive mappings of Browder-Petryshyn type. Taiwan. J. Math. 10, 87-99 (2006)

25. Takahashi, W, Toyoda, M: Weak convergence theorems for nonexpansive mappings and monotone mappings. J. Optim. Theory Appl. 118, 417-428 (2003)

26. liduka, H, Takahashi, W, Toyoda, M: Approximation of solutions of variational inequalities for monotone mappings. Panam. Math. J. 14, 49-61 (2004)

27. Xu, HK: Inequalities in Banach spaces with applications. Nonlinear Anal. 16, 1127-1138 (1991)

28. Kamimura, S, Takahashi, W: Strong convergence of a proximal-type algorithm in a Banach space. SIAM J. Optim. 13, 938-945 (2002)

29. Xu, HK: Iterative algorithms for nonlinear operators. J. Lond. Math. Soc. 66, 240-256 (2002)

30. Reich, S: Weak convergence theorems for nonexpansive mappings in Banach spaces. J. Math. Anal. Appl. 67, 274-276 (1979)

31. Aoyama, K, Kimura, Y, Takahashi, W, Toyoda, M: Approximation of common fixed points of a countable family of nonexpansive mappings in a Banach space. Nonlinear Anal. 67, 2350-2360 (2007)

32. Jung, JS: Iterative approaches to common fixed points of nonexpansive mappings in Banach spaces. J. Math. Anal. Appl. 302, 509-520 (2005)

33. Bruck, RE: Properties of fixed point sets of nonexpansive mappings in Banach spaces. Trans. Am. Math. Soc. 179 251-262 (1973)

34. Suzuki, T: Strong convergence of Krasnoselskii and Mann's type sequences for one parameter nonexpansive semigroups without Bochner integrals. J. Math. Anal. Appl. 305, 227-239 (2005)

35. Cho, YJ, Zhou, HY, Guo, G: Weak and strong convergence theorems for three-step iterations with errors for asymptotically nonexpansive mappings. Comput. Math. Appl. 47, 707-717 (2004)

10.1186/1687-1812-2013-249

Cite this article as: Ceng and Wen: System of variational inequalities and an accretive operator in Banach spaces. Fixed Point Theory and Applications 2013, 2013:249

\section{Submit your manuscript to a SpringerOpen ${ }^{\odot}$ journal and benefit from:}

- Convenient online submission

- Rigorous peer review

- Immediate publication on acceptance

- Open access: articles freely available online

- High visibility within the field

- Retaining the copyright to your article 